\title{
The repressor and co-activator HsfB1 regulates the major heat stress transcription factors in tomato
}

\author{
Sotirios Fragkostefanakis ${ }^{1}$ | Stefan Simm ${ }^{1,2}$ | Asmaa El-Shershaby ${ }^{1}$ | Yangjie $\mathrm{Hu}^{1}$ | \\ Daniela Bublak $^{1}$ | Anida Mesihovic ${ }^{1}$ | Katrin Darm ${ }^{3}$ | Shravan Kumar Mishra ${ }^{1}$ (D) | \\ Bettina Tschiersch ${ }^{4}$ | Klaus Theres ${ }^{5}$ | Christian Scharf ${ }^{3}$ | Enrico Schleiff ${ }^{1,2,6}$ (D) | \\ Klaus-Dieter Scharf ${ }^{1}$
}

${ }^{1}$ Department of Biosciences, Molecular Cell Biology of Plants, Goethe University,

Frankfurt am Main, Germany

${ }^{2}$ Frankfurt Institute of Advanced Studies (FIAS), Frankfurt am Main, Germany

${ }^{3}$ Department of Otorhinolaryngology, Head and Neck Surgery, University Medicine, Greifswald, Germany

${ }^{4}$ Leibniz Institute of Plant Biochemistry, Halle (Saale), Germany

${ }^{5}$ Max Planck Institute for Plant Breeding Research, Cologne, Germany

${ }^{6}$ Buchmann Institute for Molecular Life Sciences (BMLS), Goethe University, Frankfurt am Main, Germany

Correspondence

Enrico Schleiff and Klaus-Dieter Scharf, Department of Biosciences, Molecular Cell Biology of Plants, Goethe University, Frankfurt am Main D-60438, Germany. Email: schleiff@bio.uni-frankfurt.de; scharf@bio.uni-frankfurt.de

\section{Present Address}

Shravan Kumar Mishra, Department of Biological Sciences, Indian Institute of Science Education and Research (IISER) Mohali, Knowledge City, Sector 81, SAS Nagar, 140306 Punjab, India.

Bettina Tschiersch, BASF SE, GOI/WB - C006, D-67056 Ludwigshafen, Germany.

\section{Funding information}

Bundesministerium für Bildung und Forschung; China Scholarship Council; Deutsche Forschungsgemeinschaft, Grant/ Award Number: SFB902; Deutscher Akademischer Austausch Dienst Kairo; Marie Curie Initial Training Network SPOT-ITN

\begin{abstract}
Plants code for a multitude of heat stress transcription factors (Hsfs). Three of them act as central regulators of heat stress (HS) response in tomato (Solanum lycopersicum). HsfA1a regulates the initial response, and HsfA2 controls acquired thermotolerance. HsfB1 is a transcriptional repressor but can also act as co-activator of HsfA1a. Currently, the mode of action and the relevance of the dual function of HsfB1 remain elusive. We examined this in HsfB1 overexpression or suppression transgenic tomato lines. Proteome analysis revealed that HsfB1 overexpression stimulates the co-activator function of HsfB1 and consequently the accumulation of HS-related proteins under non-stress conditions. Plants with enhanced levels of HsfB1 show aberrant growth and development but enhanced thermotolerance. HsfB1 suppression has no significant effect prior to stress. Upon HS, HsfB1 suppression strongly enhances the induction of heat shock proteins due to the higher activity of other HS-induced Hsfs, resulting in increased thermotolerance compared with wild-type. Thereby, HsfB1 acts as co-activator of HsfA1a for several Hsps, but as a transcriptional repressor on other Hsfs, including HsfA1b and HsfA2. The dual function explains the activation of chaperones to enhance protection and regulate the balance between growth and stress response upon deviations from the homeostatic levels of HsfB1.
\end{abstract}

\section{KEYWORDS}

heat stress transcription factor, heat shock protein, proteome, Solanum lycopersicum, stress response, thermotolerance, tomato, transcription 


\section{1 | INTRODUCTION}

Heat stress (HS) response (HSR) mechanisms include signal perception, activation of HS transcription factors (Hsfs), and production of heat shock proteins (Hsps). All events are a prerequisite for protection from stress and maintenance of protein homeostasis (Qu, Ding, Jiang, \& Zhu, 2013). At the organismic level, HSR leads to reduced growth, alterations in the timing of developmental transitions, and anomalies in developmental programs including impaired male and female gametogenesis (Bokszczanin \& Fragkostefanakis, 2013; Lavania, Dhingra, Siddiqui, Al-Whaibi, \& Grover, 2015; Mesihovic, lannacone, Firon, \& Fragkostefanakis, 2016).

The activation of HSR is mediated by the activity of Hsfs (Scharf, Berberich, Ebersberger, \& Nover, 2012; Ohama, Sato, Shinozaki, \& Yamaguchi-Shinozaki, 2016). Plant Hsfs are categorized in classes $A, B$, and $C$ based on structural peculiarities in the DNA binding domain (DBD) and oligomerization domain (OD) and the presence of class-specific motifs (Nover et al., 2001; Scharf et al., 2012). Class A Hsfs are characterized by a specific C-terminal activator domain conferring transcriptional activator function (Döring et al., 2000; Mishra et al., 2002). Class B Hsfs harbour a conserved amino acid motif LFGV in their CTD discussed to confer repressor function (Bharti et al., 2004; Czarnecka-Verner, Pan, Salem, \& Gurley, 2004; Ikeda \& Ohme-Takagi, 2009), whereas class $C$ has neither activation nor repressor domains.

In tomato, the onset of the response requires the activity of HsfA1a, which is constitutively expressed, but remains inactive under non-stress conditions by interaction with cytosolic Hsp70 and Hsp90 chaperones (Hahn, Bublak, Schleiff, \& Scharf, 2011; Mishra et al., 2002). In Arabidopsis thaliana, the master regulator function is shared among the four members of the HsfA1 subfamily (Liu, Liao, \& Charng, 2011). Upon onset of stress response, additional Hsfs are induced such as HsfA1b, HsfA2, and HsfA3 to allow stress signal amplification and establishment of thermotolerance and stress memory as shown in A. thaliana and Solanum lycopersicum (Bharti et al., 2000; Charng et al., 2006; Giorno et al., 2010; Kumar et al., 2009; Lämke, Brzezinka, Altmann, \& Bäurle, 2016; Scharf et al., 1998; Schramm et al., 2008; Yoshida et al., 2011).

In tomato, HsfA2 is deposited to cytosolic HS granules during HS upon interaction with small Hsps (sHsps; Port et al., 2004). Upon several cycles of HS or during long-term stress, HsfA2 is released from the HS granules and interacts with HsfA1a to facilitate the stronger transcriptional activity required for protection of protein homeostasis (Chan-Schaminet, Baniwal, Bublak, Nover, \& Scharf, 2009; Fragkostefanakis et al., 2014, 2016; Scharf et al., 1998).

In contrast to the established activator function of several HsfAs, the role of class B Hsfs is less understood. In A. thaliana, HsfB1 and HsfB2b act as repressors of HSR genes in non-stressed cells and during recovery from stress (Ikeda, Mitsuda, \& Ohme-Takagi, 2011). $h s f b 1$-hsfb2b knock-out mutants show increased basal thermotolerance (BTT) compared with wild-type (WT) due to induction of HS-related genes and exhibit also higher disease resistance due to enhanced levels of defence-related genes (Ikeda et al., 2011; Kumar et al., 2009). Furthermore, $h s f b 1-h s f b 2 b$ mutants exhibit reduced acquired thermotolerance (ATT) due to the weaker induction of HS-related genes (Ikeda et al., 2011). However, the mechanism by which HsfB members $A$. thaliana can have a stimulatory effect on transcription of HS-responsive genes remains unknown as only a direct repressor function has been reported yet.

Tomato HsfB1 acts as a repressor of HS-inducible genes but as a co-activator of HsfA1a and other non-Hsf transcription factors (Bharti et al., 2004). The latter has been concluded from GUS reporter assays in tobacco protoplasts, in which tomato HsfB1 was shown to co-activate promoters of $A$. thaliana housekeeping genes including actin and $\mathrm{Hsc70}$ (Bharti et al., 2004). Consequently, detailed information on the role of HsfB1 in the regulation of stress responsive networks and particularly on the endogenous tomato system are still lacking.

The activity of tomato HsfB1 is controlled by feedback mechanisms, which regulate the protein levels in a condition- and functiondependent manners (Röth, Mirus, Bublak, Scharf, \& Schleiff, 2016). The strict regulation is essential for proper cellular surveillance as, for example, enhanced expression of $A$. thaliana HsfB1 induces cell death in tobacco leaves (Zhu, Thalor, Takahashi, Berberich, \& Kusano, 2012). Tomato HsfB1 is produced under non-stress conditions, but the protein is rapidly degraded via the $26 \mathrm{~S}$ proteasome (Hahn et al., 2011). The latter requires the interaction of HsfB1 with Hsp90 and binding to promoter elements (Hahn et al., 2011). Moreover, HsfB1 is rapidly degraded when acting as repressor in a repressor domaindependent manner (Röth, Mirus, Bublak, Klaus-Dieter, \& Schleiff, 2016). Under HS, HsfB1 is stabilized by recruitment into activator complexes with HsfA1a (Röth et al., 2016).

Summarizing, on the one hand, a direct correlation of HsfB1 mode of action between A. thaliana and tomato is not possible due to the seemingly functional differences between the two proteins. On the other hand, HsfB1 function proposed from Bharti et al. (2004) provides only a general model for function. Consequently, neither the array of genes and proteins affected by HsfB1 activity are known nor the physiological relevance of HsfB1 mode of action is understood.

We addressed the mode of the repressor and co-activator function of HsfB1 by analysing transgenic tomato plants with ectopic or suppressed levels of HsfB1. The ectopic accumulation of HsfB1 under non-stress conditions alters growth and development. Moreover, overexpression as well as suppression of HsfB1 leads to increased seedling thermotolerance and results in the activation of protection mechanisms for photosystems in young leaves. We observed that HsfB1 manipulation alters the abundance of proteins involved in various biological processes including several with putative housekeeping functions, such as cognate Hsps, translation elongation factors, and proteasome-related proteins. In addition, we show that the co-activator function of HsfB1 is not general for all HS-induced genes, as HsfB1 acts as repressor on promoters of stress induced Hsfs, such as HsfA2 and HsfA1b. Consequently, suppression of HsfB1 reliefs the repression on these Hsfs, which in turn leads to a stronger response under HS and thereby increased thermotolerance. We conclude that the deviation from the homeostatic levels of HsfB1 activate gene networks to ensure adequate response and protection under high temperatures. Our results shed light to the complex role of the bifunctional HsfB1 and give further insights into the regulation of HSR in an important crop. 


\section{2 | MATERIALS AND METHODS}

\section{1 | Generation of HsfB1 transgenic plants}

S. lycopersicum plants (cv. Moneymaker) were transformed using the Agrobacterium tumefaciens strain GV3101(pMP90) (Koncz \& Schell, 1986) as described for HsfA1a transgenic plants (Mishra et al., 2002). In brief, the $\beta$-glucuronidase (uid A) gene was removed from the binary vector pGPTV-KAN (Becker et al., 1992), followed by insertion of a DNA fragment containing the sense or antisense Solanum peruvianum HsfB1 cDNA expression cassette from the corresponding pRT vectors under the control of CaMV $35 \mathrm{~S}$ promoter to generate $B 1 O E$ (overexpression) and B1AS (antisense) transgenic lines, respectively (Scharf, Rose, Zott, Schöff, \& Nover, 1990). T4 generation plants were used for analyses, derived from T3 homozygous plants, based on segregation analysis tests.

\section{2 | Heat stress treatment}

Eight-week-old WT and HsfB1 transgenic tomato plants grown in a glasshouse under a $16 / 8 \mathrm{hr}$ day/night cycle $\left(25-20^{\circ} \mathrm{C}\right)$ were trans-

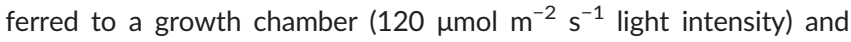
exposed to $\mathrm{HS}$ for $1 \mathrm{hr}$ at $39^{\circ} \mathrm{C}$ and then allowed to recover for $1.5 \mathrm{hr}$ at $25^{\circ} \mathrm{C}$ or kept for the same time at $25^{\circ} \mathrm{C}$ as control. Each biological replicate was consisted by young leaves or stems pooled from at least eight control and stressed plants, respectively. HS in all cases was applied around 11-12:00 a.m.

\section{3 | Thermotolerance assays}

BTT and ATT were examined in 4-day-old seedlings of WT and all transgenic lines as described previously (Fragkostefanakis et al., 2016). Thermotolerance in relation to photosynthetic capacity was investigated in plants grown on soil in a glasshouse under long day (16/8 hr, $25 / 20^{\circ} \mathrm{C}$, day/night) conditions.

\section{4 | Pulse amplitude modulated fluorometry measurements}

The photosynthetic activity of control and stressed young and old leaves was estimated by pulse amplitude modulated (PAM) fluorometry (Walz). Six-week-old plants were transferred to a climate chamber with artificial illumination at $120 \mu \mathrm{mol}$. HS treatments were performed during daytime by a gradual increase of the temperature from $25^{\circ} \mathrm{C}$ to $43^{\circ} \mathrm{C}$ within $90 \mathrm{~min}$. After additional $90 \mathrm{~min}$ at $43^{\circ} \mathrm{C}$, temperature setting was returned to $25^{\circ} \mathrm{C}$ to allow slow cooling down of the chamber. The HS regime was repeated daily for the subsequent 3 days before the return of the plants to the glasshouse for an additional period of 5 days for recovery. Photosynthetic activities were followed by PAM measurements on isolated leaflets from younger and older leaves, respectively, after dark adaptation for $30 \mathrm{~min}$. Effective photochemical quantum yield of the photosystem II (Ф (II)), quantum yield of lightinduced non-photochemical fluorescence quenching (Ф (NPQ)), effective photochemical quantum yield of open photosystem II centers (Ф (II)/qL), and electron transport rate of the photosystem II (ETR) were determined according to the instructions of the manufacturer.
Five different areas from each young and old leaf were measured (technical replicates), and for each genotype, three different plants were used. The experiment was repeated three independent times.

\section{5 | RNA extraction and qRT-PCR}

Total RNA was extracted using the E.Z.N.A. Plant RNA Kit (Omega Bio-Tek, Norcross, GA, USA) following the manufacturer instructions. One microgram of total RNA was used for cDNA synthesis with Revert Aid reverse transcriptase (Thermo Scientific) following the manufacturer's protocol. Expression of selected genes was determined using quantitative real-time PCR ( $q R T-P C R$ ) on a Stratagene Mx3000P cycler (Agilent Technologies). The reaction (10 $\mu \mathrm{l})$ consisted of gene primers (Dataset S1), PerfeCTa ${ }^{\circledR}$ SYBR ${ }^{\circledR}$ Green FastMix Low ROX $^{\mathrm{TM}}$ (Quanta Biosciencies) and the template. Thermal cycling conditions were $95^{\circ} \mathrm{C} / 3$ min followed by $95^{\circ} \mathrm{C} / 15 \mathrm{~s}, 60^{\circ} \mathrm{C} / 30 \mathrm{~s}$, and $72^{\circ} \mathrm{C} / 30 \mathrm{~s}$ for 40 cycles. Gene primers were designed using PRIMER3 (www-genome.wi.mit.edu/cgi-bin/primer/primer3.cgi/). Data were analysed by standard methods (Livak \& Schmittgen, 2001) and presented as relative levels of gene expression using EF1a (Solyc06g005060) gene as internal standard.

\subsection{Two-dimensional differential in gel electrophoresis (2D-DIGE)}

Total protein extracts from young leaves and stem sections of the youngest internodes were prepared as previously described (Mishra et al., 2002) and precipitated with $80 \%$ acetone. After successive washing steps in $80 \%$ acetone and $70 \%$ ethanol, protein precipitates were dissolved in $8 \mathrm{M}$ urea/2 $\mathrm{M}$ thiourea solution. Protein concentration was determined by the Bradford assay (Bradford, 1976). Quantified protein extracts were minimally labelled using the Refraction2D ${ }^{\mathrm{TM}}$-kit (NH DyeAGNOSTICS GmbH, Halle/S., Germany). Aliquots corresponding to $50 \mathrm{\mu g}$ of protein were labelled with $400 \mathrm{pmol}$ of either G-200 or G-300 dyes, in which labelling of all samples was done by dye swapping. As an internal standard, aliquots of all individual samples were pooled and labelled with G-100.

Linear pH 3-10, 24 cm IPG strips (GE Healthcare) were rehydrated overnight in rehydration solution containing two labelled samples (G-200 and G-300, each $50 \mu \mathrm{g}$ ) and the corresponding internal standard (G-100, $50 \mu \mathrm{g}$ ) for first dimension separation. Isoelectric focusing (IEF) was performed using a Multiphor II apparatus (GE Healthcare). The second dimension separation of proteins was done on $12.5 \%$ SDS-polyacrylamide gels (Nishtala et al., 2013; Thiele et al., 2007).

\section{7 | Image analysis and statistical tests}

After separation in the second dimension, the gel images were recorded on a Typhoon 9400 Scanner (GE Healthcare) and analysed with Delta2D software version 4.6 (Decodon, Greifswald, Germany; Hammer et al., 2010). After gel matching, spot volumes were analysed by Delta2D and the TMEV (Multi Experiment Viewer) statistic module (Howe, Sinha, Schlauch, \& Quackenbush, 2011). Statistically significant differences in spot intensities among the groups consisting of two replicates each were calculated by oneway ANOVA applying Welch's $t$ test. 


\section{8 | In-gel digestion of proteins and mass spectrometry}

Preparative 2D gels were run each with $550 \mu \mathrm{g}$ of the pooled leaf and stem samples, respectively. Protein spots were visualized by colloidal coomassie staining (Thiele et al., 2007), and images were recorded and aligned with those of analytical gels in Delta2D. Spots of interest were processed for identification as described by Eymann et al. (2002). Briefly, tryptic digestion was performed automatically in an Ettan Spot Handling Workstation (Amersham Biosciences) as well as the subsequent spotting of peptide solution onto MALDI targets. Samples were measured using the Matrix-Assisted Laser Desorption-lonization Time-of-Flight (MALDI-TOF-TOF) instrument (Proteome-Analyser 4800; Applied Biosystems, Foster City, CA, USA) as described previously (Lendeckel et al., 2015). Peak lists from combined MS and MS/MS measurements were compared with a UniProt-SwissProt database (Rel. 51.5 restricted to human taxonomy) by the MASCOT search engine Version 2.1. Protein identifications and statistically relevant data were combined via unique spot-IDs using the MSRepo database software (Decodon, Greifswald, Germany).

\section{9 | LC-MS/MS analysis}

Aliquots of protein extracts corresponding to $4 \mu \mathrm{g}$ of total protein were digested overnight at $37^{\circ} \mathrm{C}$ with trypsin (Promega) after protein reduction and alkylation. Tryptic peptides were desalted with C-18 ZipTip (Millipore) following the manufacturer's instructions. LC-MS/MS measurements were done as described before (Palm et al., 2016), including reverse phase separation of tryptic peptides performed on a Proxeon Easy nano-LC system and mass spectrometric analysis using the Orbitrap Velos MS. Peptides were identified by an automated SORCERER/SEQUEST search (Sorcerer built 4.04, Sage-N Research Inc., Milpitas, CA, USA) against a S. lycopersicum database generated from ITAG 2.4 (https://www.solgenomics.net/). Only proteins with at least two significant peptides (greater than $95.0 \%$ probability) were considered for identification and Sequest identifications required at least XCorr scores of greater than 2.5 for double and 3.0 for triple charged ion (Keller, Nesvizhskii, Kolker, \& Aebersold, 2002; Nesvizhskii, Keller, Kolker, \& Aebersold, 2003).

\subsection{0 | Principal component analysis (PCA) and k-means clustering}

All identified spots via 2D-DIGE for the different proteome samples were used for PCA and $k$-means clustering. The FactoMineR package (version 1.31.4; http://factominer.free.fr/) was used to perform the PCA (Lê, Josse, \& Husson, 2008) using RStudio (https://www. rstudio.com/). The three main principal components (PCs) were used for separating the single samples. For each PC, the percentage of partitioning of all spots among the samples was calculated.

$k$-means clustering was performed by Multiple experiment Viewer tool (MeV version 4.9.0; https://sourceforge.net/projects/mev-tm4/). The number of clusters $(k)$ was chosen based on a 50 times Figure Of Merit (FOM). The LC-MS/MS and DIGE values were transformed in relative abundance of each leaf and stem datasets. Each $k$-means clustering of each approach was performed 25 times, and the result with the lowest variance was chosen. For cluster comparison, Euclidean hierarchical clustering of mean cluster values was performed by $\mathrm{MeV}$. Functional categorization of selected proteins was done using MapMan (http://mapman.gabipd.org/) using the mapping file for S. lycopersicum ITAG2.3 (http://mapman.gabipd.org/de/mapmanstore).

\subsection{Protein analysis by immunodetection}

For immunoblot analysis, frozen leaves (50-100 mg) were used for protein extraction as previously described (Mishra et al., 2002); 15-20 $\mu \mathrm{g}$ of protein extracts were mixed with an equal volume of $2 \times$ SDS sample buffer and separated on $10 \%$ SDS-polyacrylamide gel. Proteins were transferred to nitrocellulose membrane (PROTRAN ${ }^{\circledR}$ Nitrocellulose Transfer Membrane, Whatman) and processed for chemiluminescence detection following the manufacturer's protocol (PerkinElmer). Hsp101 antibody was purchased from Agrisera (AS07-253).

\subsection{2 | GUS reporter assay}

Tomato (S. lycopersicum cv. Moneymaker) mesophyll protoplasts were purified and transformed by polyethylene glycol-mediated transformation as previously described (Mishra et al., 2002). Fifty thousand protoplasts were transformed with a total of $10 \mu \mathrm{g}$ of plasmid DNA per sample consisting of $0.5 \mu \mathrm{g}$ of each Hsf expressing plasmid and $1 \mu \mathrm{g}$ of the reporter construct. The total amount of plasmids was complemented with pRT-Neo mock plasmid to $10 \mu \mathrm{g}$. Upon transformation, protoplasts were incubated for $6 \mathrm{hr}$ at $25^{\circ} \mathrm{C}$. GUS activities were determined as described previously (Chan-Schaminet et al., 2009; Treuter, Nover, Ohme, \& Scharf, 1993).

Plasmids encoding for HsfA1a, HsfA2, HsfA3, and HsfB1 as well as PHsp17*::GUS have been described elsewhere (Hahn et al., 2011; Nover et al., 2001; Treuter et al., 1993). The promoter reporter constructs of HsfA1b (Solyc03g097120), HsfA2 (Solyc08g062960), HsfA3 (Solyc09g009100), Hsp70-1 (Solyc06g076020), and Hsp21.5-ER (Solyc11g020330) were generated by cloning approximately $1 \mathrm{~kb}$ fragments upstream of the start codon for each gene in the pRT-GUS vector. The expression construct of HsfA1b, HsfA6b (Solyc06g053950), and HsfA7 (Solyc09g065660) was prepared by cloning the open reading frame to the carboxyl terminus of a $3 \times \mathrm{HA}$ coding sequence under the control of CaMV $35 \mathrm{~S}$ promoter using Sall-Xbal restriction sites (Table S1). HsfA4b and HsfA9 have been described elsewhere (Baniwal, Kwan, Scharf, \& Nover, 2007; Röth et al., 2016). The levels of each protein were detected by immunoblot analysis using either anti-HA or Hsfspecific antibodies (Chan-Schaminet et al., 2009; Hahn et al., 2011).

\section{3 | RESULTS}

\section{1 | Identification of HsfB1 suppression and overexpression transgenic plants}

We generated transgenic plants with ectopic or suppressed HsfB1 expression in the background of S. lycopersicum cv. Moneymaker. The transgenic lines were produced by expression of a cDNA cassette of tomato HsfB1 (CAA39034; Scharf et al., 1990) in the sense or 
antisense (AS) orientation under the control of CaMV-35S promoter, aiming for overexpression or suppression, respectively.

The level of the endogenous HsfB1 transcripts in the transgenic lines was analysed by qRT-PCR in leaves of 6-week-old plants and compared with WT. Plants were exposed either to $39^{\circ} \mathrm{C}$ for $1 \mathrm{hr}$ (Figure 1a, sample "HS"), followed by $1.5 \mathrm{hr}$ recovery at $25^{\circ} \mathrm{C}$ (sample "HR") or kept at $25^{\circ} \mathrm{C}$ (non-treated plants; sample "C"). We selected two antisense lines (B1AS-18, AS-21) and one sense co-suppression line (B1CS-39) with significantly reduced levels of HsfB1 transcripts in control and stressed samples (Figure 1a).

Overexpression lines were identified by qRT-PCR using primers amplifying both the endogenous and the transgene mRNA. We selected three sense lines (B1OE-5, -41 and -46) with fivefold to 15-fold higher expression of HsfB1 total levels in non-stressed and stressed leaves when compared with the level in WT plants (Figure 1b). The transcript level of the endogenous HsfB1 was enhanced in the recovery sample in all three sense lines compared with WT (Figure 1a).

The impact of the antisense repression (AS), co-suppression (CS), or overexpression (OE) on HsfB1 protein level was confirmed. HsfB1 protein was barely detectable under control conditions in WT plants, strongly accumulated after treatment at $39^{\circ} \mathrm{C}$ and rapidly reduced to basal control levels after recovery (Figure 1c). Significantly reduced levels of the endogenous HsfB1 were detected in control and stressed B1AS and B1CS lines (Figure 1c). In turn, HsfB1 accumulates at higher levels in all three sense lines under all conditions when compared with WT (Figure 1c). Collectively, we identified three independent HsfB1 suppression and three overexpression lines, which were used for further investigation.

\subsection{Accumulation of HsfB1 affects plant growth and development}

Phenotypic analysis revealed that the overexpression of HsfB1 affects tomato plant growth and development, whereas suppression of HsfB1 has only minimal effects under normal growth conditions (Figure 2a). The B1OE lines have reduced stem growth compared with WT plants at 36 and 44 days post seed imbibition (dpi), whereas there is no significant difference at earlier time points (Figure 2b). No significant differences exist in seed germination or seedling early growth between the transgenic and WT plants (data not shown). Thus, the growth retardation coincides with first flower bud appearance, approximately 5 weeks dpi.

In agreement, the youngest internodes of the overexpression lines are significantly shorter compared with WT or the suppression lines (Figure 2c). Suppression of HsfB1 results in enhanced length of the older internodes; however, this does not have a significant effect on the overall stem growth, which is similar to WT (Figure 2c).

WT and suppression plants develop side shoots after transition of the primary apical shoot meristem to the formation of the first inflorescence mainly at apical internodes numbers 10-12 (Figure 2d). In contrast, B1OE lines develop adaxial side shoots at the older internodes 1-8. Although the duration of flowering time until fruit set is similar for all lines (not shown), HsfB1 overexpression leads to earlier

\section{(a)}
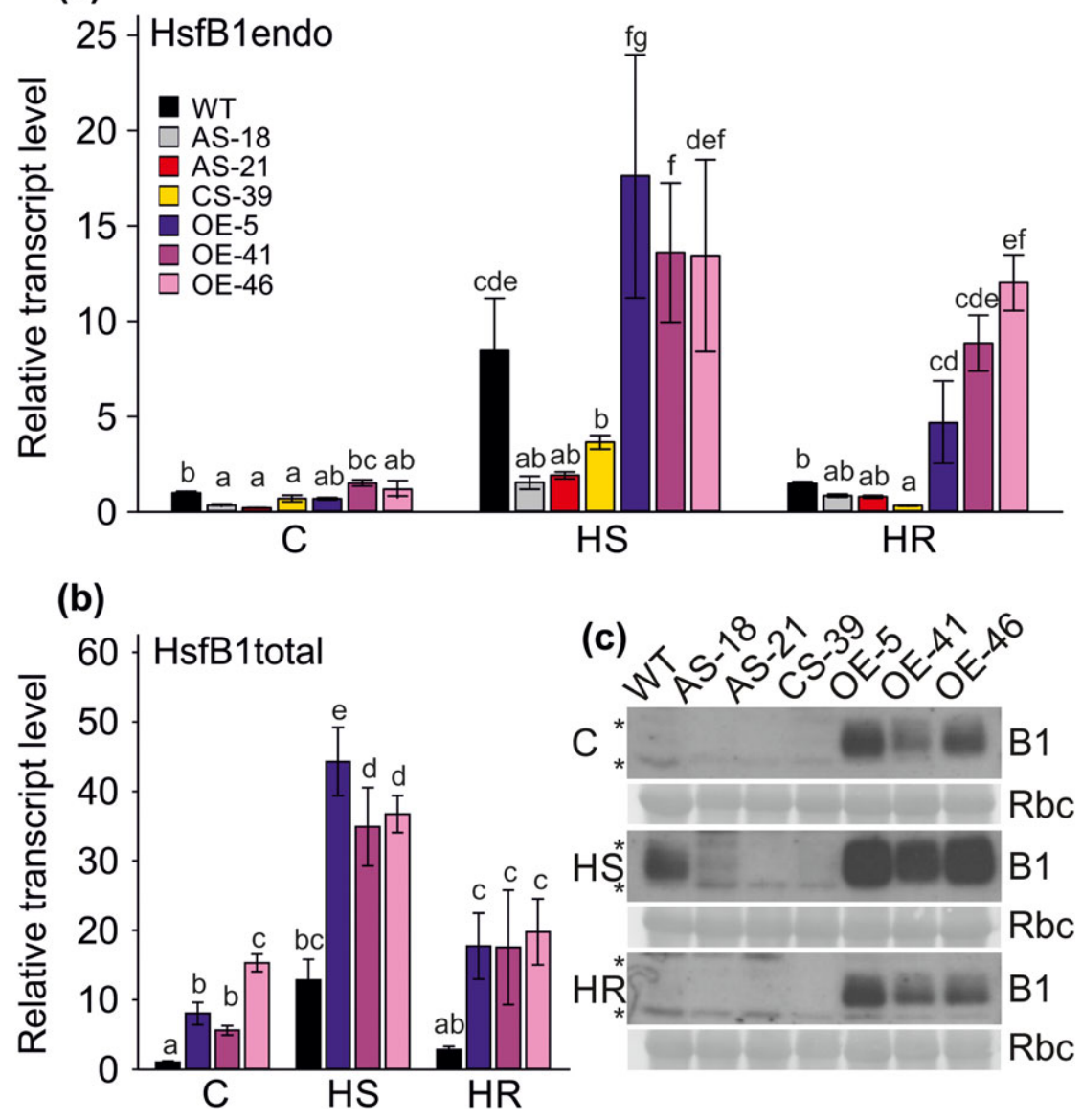

FIGURE 1 Expression of HsfB1 in leaves of sense and antisense transgenic tomato lines. Relative transcript levels of (a) endogenous and (b) endogenous and transgene HsfB1 (total) in leaves of wild-type (WT), antisense (AS), co-suppression (CS), or sense overexpression lines (OE) exposed either for $1 \mathrm{hr}$ to $39^{\circ} \mathrm{C}(\mathrm{HS})$ and subsequently $1.5 \mathrm{hr}$ to $25^{\circ} \mathrm{C}$ (HR) or for the same time at $25^{\circ} \mathrm{C}(\mathrm{C})$ were determined by qRT-PCR. EF1a was used as housekeeping gene and results were normalized to control WT sample. Bars are means of four replicates $\pm S D$. Different letters denote statistically significant difference at $p<0.05$ (ANOVA and Duncan's multiple range test). (c) Leaves of WT and transgenic plants were treated as in (a). HsfB1 levels in $40 \mu \mathrm{g}$ of total protein extract of WT and transgenic lines were determined by immunoblot analysis with an HsfB1 antibody. Ponceau S staining of large subunit of Rubisco (Rbc) is shown for loading control. Asterisks indicate cross reactivity of the antibody with non-HsfB1 proteins 
(a)
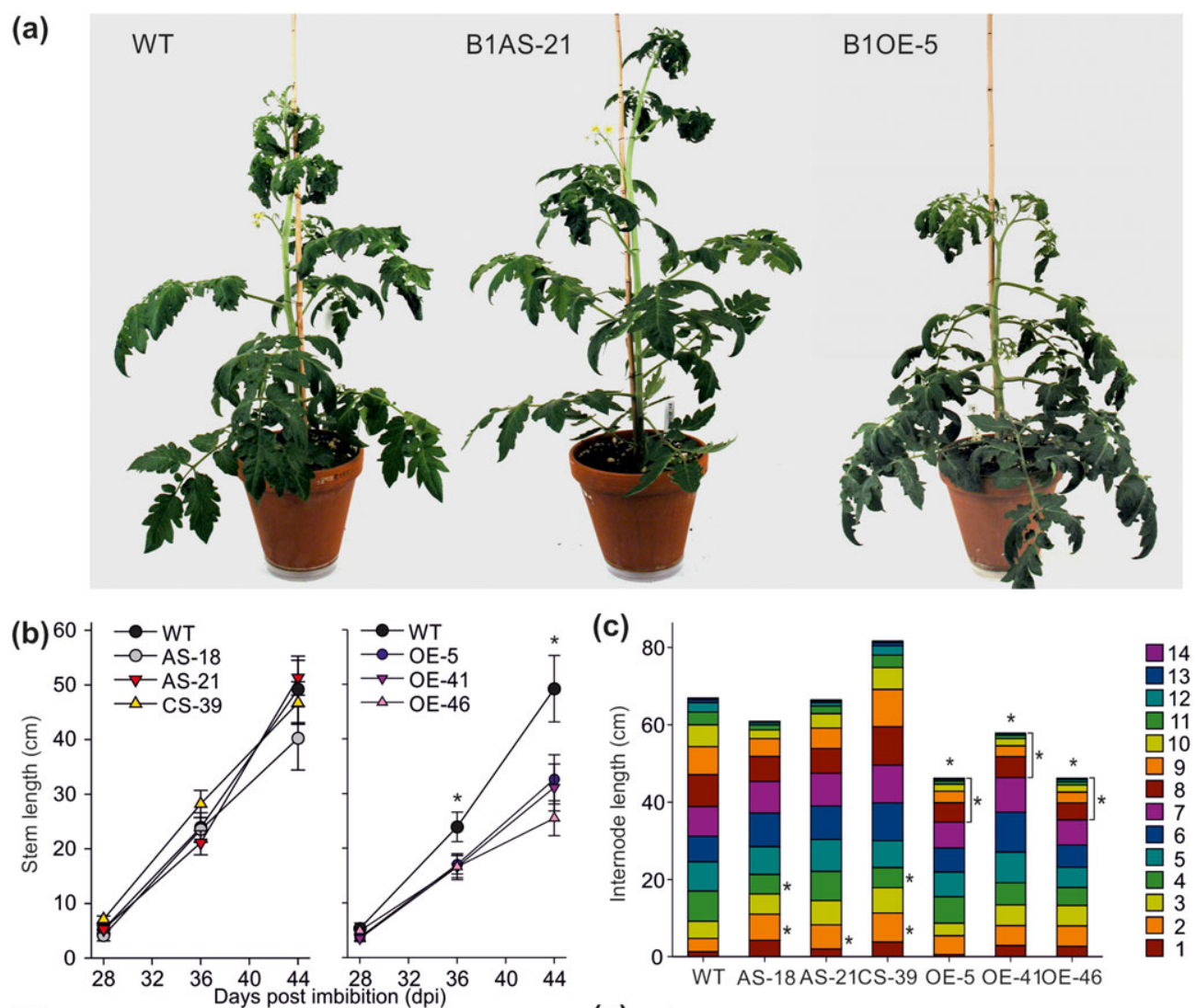

(d)

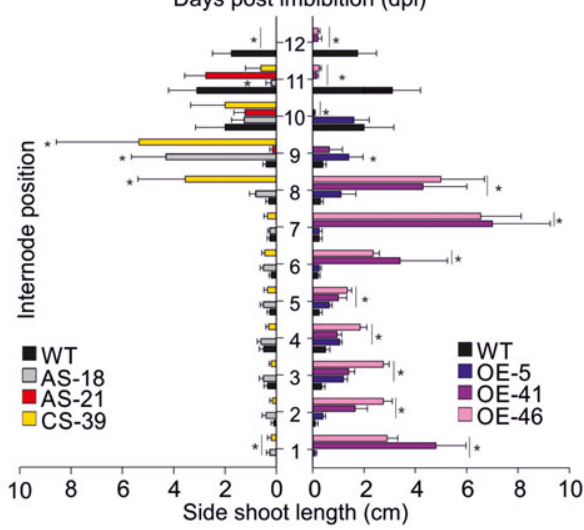

(e) $100+\square$

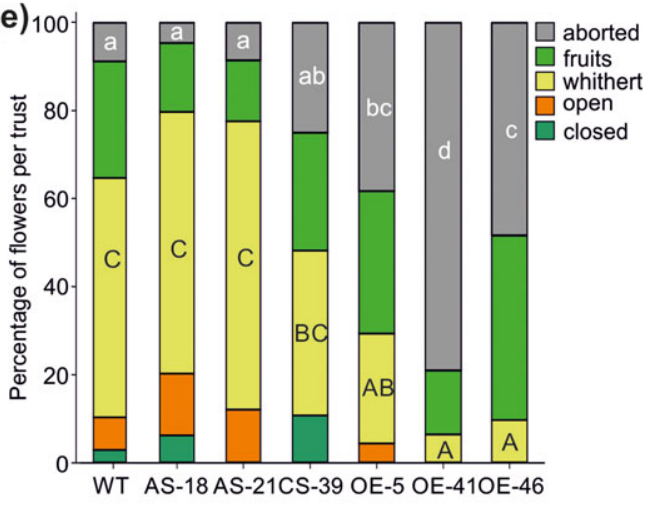

FIGURE 2 Effect of overexpression and suppression of HsfB1 on growth and development. (a) Images of representative WT, B1AS-21, and B1OE-5 plants at 65 dpi grown under standard control conditions. (b) Stem length of WT and transgenic plants 28, 36, and 44 dpi, and (c) internode length of WT and transgenic plants $52 \mathrm{dpi}$. The cumulative length of the internodes is the average stem length $52 \mathrm{dpi}$. Asterisks indicate significant difference between transgenic and WT plants for the respective internode (right side of bar) or stem length (top; $p<0.05$ ). (d) Length of side shoots at each internode of WT and transgenic plants. Each data point is the average $\pm S D$ of at least 10 plants grown under standard conditions from one representative experiment. (e) Percentage of flowers at pre-anthesis (closed), anthesis (open), post-anthesis (withered petals), flowers with fruit set or aborted flowers on the first inflorescence $65 \mathrm{dpi}$. Different letters denote statistically significant difference at $p<0.05$, as indicated by ANOVA and Duncan's multiple range test

fruit set of approximately 1 week compared with WT (Figure 2e), which coincides with the observed growth retardation. We also detected a higher frequency of flower or fruit abortion in HsfB1 overexpression lines compared with the WT plants (Figure 2e). Collectively, HsfB1 overexpression leads to retarded plant growth until the reproductive period of the plant and to defects in flowering and fruit set. Suppression of HsfB1 does not yield notable phenotypic differences with the exception of the longer basal internodes. We exclude the possibility that the phenotype is due to the transformation event itself, as on the one hand the transgenic lines derived from independent transformation events whereas on the other hand the co-suppression transgenic line did not show any defects on growth or development (Figure 2).

\section{3 | Effect of HsfB1 expression level manipulation on basal and acquired thermotolerance}

The importance of HsfB1 in tomato BTT and ATT was determined by relative hypocotyl elongation of seedlings during the recovery period after a HS treatment (Figure 3). Four-day-old dark grown seedlings were exposed to $42.5^{\circ} \mathrm{C}$ or $45^{\circ} \mathrm{C}$ for $1.5 \mathrm{hr}$ followed by 4 days of recovery in darkness. Both HS treatments caused a reduction in 
(a)

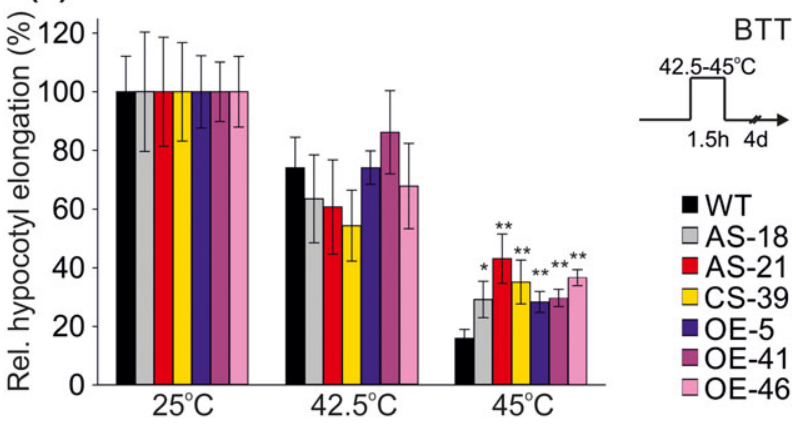

(b)

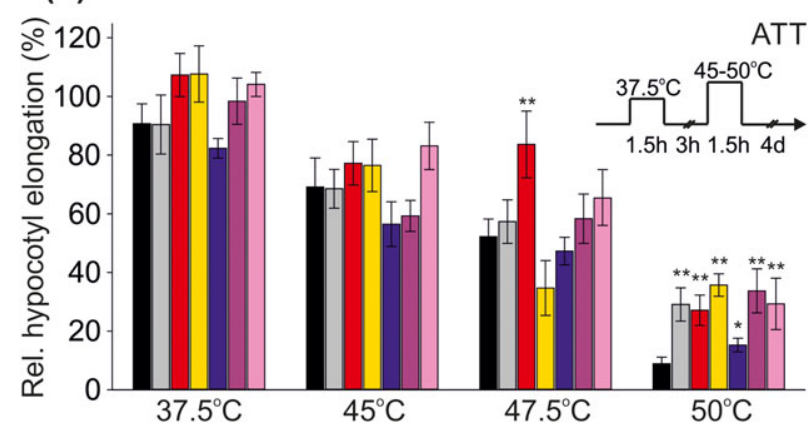

FIGURE 3 Effect of HsfB1 overexpression or suppression on thermotolerance of tomato seedlings. (a) Four-day-old seedlings were exposed to $42.5^{\circ} \mathrm{C}$ or $45^{\circ} \mathrm{C}$ for 1 hour, followed by determination of hypocotyl elongation relative to seedlings at control conditions $\left(25^{\circ} \mathrm{C}\right)$ after 4 days (BTT). (b) Four-day-old seedlings were pretreated at $37.5^{\circ} \mathrm{C}$ for $1 \mathrm{hr}$, incubated at $25^{\circ} \mathrm{C}$ for $3 \mathrm{hr}$ and then exposed to the indicated temperatures for $1.5 \mathrm{hr}$ (ATT).

Thermotolerance is expressed as hypocotyl elongation after 4 days relative to seedlings exposed to $37.5^{\circ} \mathrm{C}$. The pictographs indicate the treatments used. Bars are means of 8-10 seedlings per genotype \pm SE. Asterisks indicate significant differences to untreated seedlings of the respective genotype compared with WT (pairwise $t$ test analyses: $\left.{ }^{*} p<0.05,{ }^{* *} p<0.01\right)$

relative hypocotyl elongation of WT seedlings (Figure 3a). Both, B1OE and HsfB1 suppression lines, show similar reduction in hypocotyl elongation after treatment at $42.5^{\circ} \mathrm{C}$, but an enhanced recovery compared with WT seedlings after $\mathrm{HS}$ at $45^{\circ} \mathrm{C}$ (Figure 3a).

For ATT measurements, seedlings were pretreated at $37.5^{\circ} \mathrm{C}$ for $1 \mathrm{hr}$, followed by 3 -hr recovery at $25^{\circ} \mathrm{C}$, and a 1.5 -hr treatment at different temperatures between $45^{\circ} \mathrm{C}$ and $50^{\circ} \mathrm{C}$. Direct exposure to $47.5^{\circ} \mathrm{C}$ or $50^{\circ} \mathrm{C}$ was lethal for all lines (not shown). After 4 days of growth, WT and transgenic lines treated at $45^{\circ} \mathrm{C}$ or $47.5^{\circ} \mathrm{C}$ did not show significant differences (Figure $3 b$ ). Only B1AS-21 exhibits an enhanced ATT at $47.5^{\circ} \mathrm{C}$. In contrast, all transgenic lines exhibit a significantly increased hypocotyl elongation in response to $50^{\circ} \mathrm{C}$. These results show that both overexpression and suppression of HsfB1 lead to increased BTT and ATT in seedlings exposed to the higher temperature regimes.

\subsection{Alteration of HsfB1 levels enhances the protection of photosystems under heat stress}

The observed phenotype of the transgenic lines with respect to growth behaviour and enhanced thermotolerance prompted the determination of the photosynthetic capacity by pulse amplitude modulation (PAM) measurements (Figures 4 and S1). For that six-week-old WT, B1OE-5 and B1AS-21 plants as representative transgenic lines were exposed to $43^{\circ} \mathrm{C}$ for $1.5 \mathrm{hr}(\mathrm{HS})$ or kept under control conditions (C). At the stage of the treatment, B1AS plants were phenotypically similar to WT, whereas B1OE plants were slightly shorter as described above (Figure 2). We analysed the effective photochemical quantum yield of the photosystem II (Ф (II)), the quantum yield of light-induced nonphotochemical fluorescence quenching ( $($ (NPQ), the effective photochemical quantum yield of open photosystem II centers ( $\left.\Phi(\mathrm{II}) / \mathrm{q}_{\mathrm{L}}\right)$, and the maximal electron transport rate of the photosystem II $\left(E T R_{\text {max }}\right)$. To address developmental effects, we measured old (OL; fourth fully emerged leaf) and young leaves (YL, youngest fully emerged leaf) individually.

The $\Phi$ (II) of B1AS-OL, but not B1OE-OL, was reduced when compared with WT in both control and stressed plants (Figure S1a). Similarly, the $\Phi(\mathrm{II}) / \mathrm{q}_{\mathrm{L}}$ in old leaves was not influenced by HS irrespective of the genetic background, but was generally lower in the B1AS and higher in the B1OE lines when compared with WT (Figure 4a, OL). In young leaves, $\Phi$ (II) was only reduced in the B1AS line after HS (Figure S1a). The comparison of the $\Phi(\mathrm{II}) / \mathrm{q}_{\mathrm{L}}$ revealed a similar yield

(a)

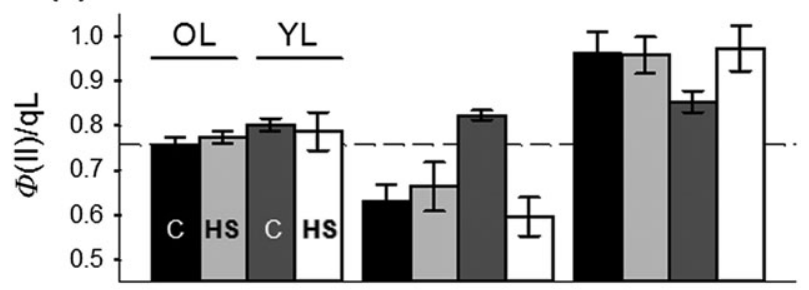

(b)

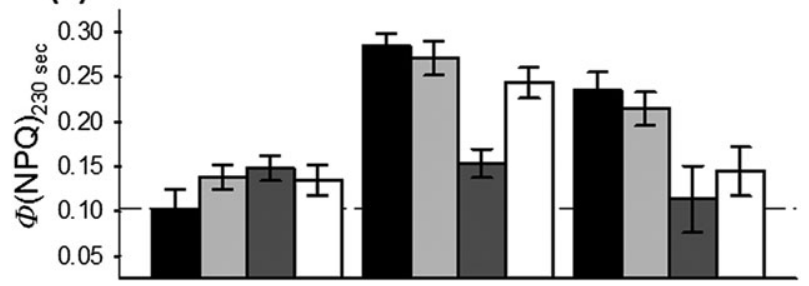

(c)

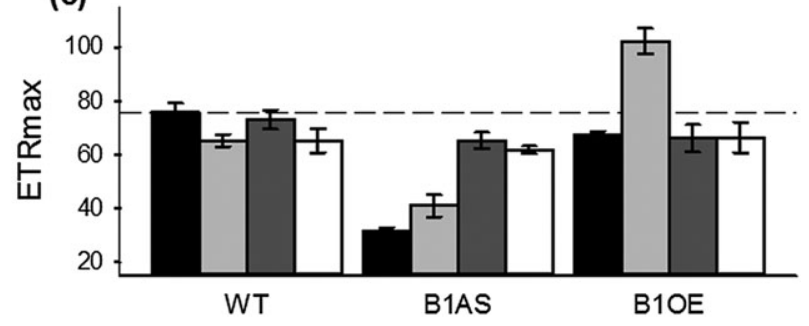

FIGURE 4 HsfB1 dependence of performance of the photosystems II. (a) The effective photochemical quantum yield of open PSII centers $\Phi(I I) / q_{L}$, (b) the quantum yield of light-induced nonphotochemical fluorescence quenching $\Phi(\mathrm{NPQ})$ after $230 \mathrm{~s}$, and (c) the maximal relative electron transfer rates (ETRmax; in $\mu \mathrm{mol} \mathrm{m} \mathrm{m}^{-2} \mathrm{~s}^{-1}$ ) for WT, B1AS-21, and B1OE-5 determined for old (OL) or young leaves $(\mathrm{YL})$ before $(\mathrm{C})$ and after heat treatment $(\mathrm{HS})$. The dashed line indicates the level of the $\mathrm{C}-\mathrm{OL}$ sample. The results are the average of three independent experiments each consisted of three plants for each genotype and five technical replicates 
for all three lines before treatment, but a reduction in the B1AS and increase in the B1OE line after HS was observed (Figure 4a, YL-HS).

The B1AS-OL and B1OE-OL have enhanced NPQ (Figures $4 \mathrm{~b}$ and $\mathrm{S} 1 \mathrm{~b})$. This suggests that an induction of protective mechanisms exists in old leaves of both transgenic lines. In young leaves, this protection was not present, but was activated in the B1AS line after heat treatment (Figure $4 \mathrm{~b}$ ). The relative ETR (Figure S1c) and the maximal relative ETR (Figure 4c) in young leaves were largely comparable between all lines and were not affected by the heat treatment. In old leaves, the ETR was reduced in the B1AS line, whereas the rate was enhanced in the B1OE line after HS (Figure 4c).

Summarizing, the performance of the photosystem II in $\mathrm{OL}$ is HsfB1-dependent as reflected by the $\Phi(\mathrm{II}) / \mathrm{q}_{\mathrm{L}}$, whereas in young leaves, the photosynthetic performance appears independent of HsfB1. In turn, HS uncovers the impact of HsfB1 for adaptation of the photosynthetic performance, as HS reduces the $\Phi$ (II) in the AS line.

\subsection{Alterations in protein abundance related to HsfB1 expression level manipulation}

HsfB1 overexpression and suppression have positive effects on seedling BTT and ATT and enhance the NPQ in response to HS. Consequently, we performed a quantitative 2D-DIGE analysis using protein extracts from young leaves and stems of 6-week-old WT, B1AS-21, and B1OE-5 plants to identify alterations in abundance of proteins that are affected by manipulation of HsfB1 activity. We investigated plants exposed to $39^{\circ} \mathrm{C}$ for $1 \mathrm{hr}$, which was followed by 1.5 -hr recovery at $25^{\circ} \mathrm{C}$ (Figure 5a; sample HR) or kept for the same time at $25^{\circ} \mathrm{C}$ as control (C). We identified in total 1712 protein spots in all samples that were quantified (Dataset S1) and excised for protein identification by mass spectrometry (Dataset S2). Out of all, 478 spots were assigned to a single gene Solyc ID and 252 spots to more than one gene (Figure S2; Dataset S2).

PCA analysis using quantified and normalized values from all spots resulted in a separation of stems and leaves by the first component representing $61 \%$ of the information (PC1; Figure 5a,b). For leaves, a distinction between WT and B1OE or B1AS lines for control and HS samples is represented by PC2 (15.6\%; Figure 5a). PC3 (8\%) showed a separation between WT or B1AS and B1OE control samples for leaves and stem (Figure $5 \mathrm{~b}$ ), between HS-treated WT, AS, and B1OE leaf samples, as well as between HS-treated WT or B1OE and B1AS stem sample.

PC2 and PC3 suggest that B1AS stem samples before and after HS behaves like WT stem control, whereas B1OE shows characteristics of the HS-treated WT-stem sample (Figure 5a,b). For leaves, this conclusion holds partially true for PC3, but not for PC2. However, in PC2 and PC3, the separation of control and HS samples is most pronounced for WT when compared with the transgenic lines. Calculating the ratio of intensity between the HS and control samples for each spot in each genotype shows that more proteins retained at steady levels in leaves and stems of transgenic lines compared with WT (Figure S3). This supports the notion for a weaker stress response regarding proteome changes.
For a more detailed picture of the protein changes, we performed hierarchical $k$-means cluster analysis individually for leaf and stem samples using the quantitative data from all spots (Figure 5c; Tables S1 and S2). The analysis was done individually for leaves and stems as these two tissues showed distinct profiles in PCA (Figure 5a,b). Clusters 1-7 contained spot IDs (533 for leaves and 282 for stems; Figure $5 c$ ) representing proteins with enhanced or reduced levels in at least one sample. In turn, clusters 8-10 (1179 spots for leaves "L" and 1420 for stems "S") showed an overall steady protein level (Dataset S2). The clusters 7L, 7S, and 4S represent proteins with a profile found only in the according tissue. Moreover, leaf and stem clusters with comparable profile largely contained different spot IDs, suggesting a tissue-specific regulation by HsfB1 for different proteins (Figure 5c; Dataset S2).

Spot IDs in clusters $1 \mathrm{~L}$ and $1 \mathrm{~S}$ represent proteins with highest abundance in WT under control conditions, but generally lower abundance in B1AS and B1OE samples compared with WT control (Figure 5c). Clusters 4L, 5L, and $5 \mathrm{~S}$ represent proteins with highest abundance in WT upon HS, whereas the abundance in the transgenic lines after HS remained comparable to WT control. However, proteins in $5 \mathrm{~S}$ are somewhat more abundant in B1OE under normal conditions.

The clusters $2 \mathrm{~L}$ and $2 \mathrm{~S}$ contain proteins with high levels in control B1AS samples only, and $6 \mathrm{~L}$ and $6 \mathrm{~S}$ represent proteins with highest abundance in B1AS after HS (Figure $5 \mathrm{c}$ ). Proteins with highest abundance in B1OE (i) in general or (ii) under control conditions are represented by clusters (i) $3 \mathrm{~L}$ and $3 \mathrm{~S}$ or (ii) $7 \mathrm{~S}$. Cluster $7 \mathrm{~L}$ unifies proteins with highest abundance in B1OE and B1AS leaves after HS (Figure 5 c). Proteins unified in cluster $4 \mathrm{~S}$ show a high abundance in WT and B1AS only under control conditions, whereas they are only high abundant in B1OE after HS. Thus, it is the only cluster where WT and AS show a comparable behaviour, whereas the situation in B1OE is contrasting.

Next, we aimed to identify proteins that are putatively related to the observed growth and thermotolerance phenotypes (Figure 6a, left). We directly compared the abundance of individual proteins in transgenic lines and WT under control conditions. A low number of proteins is affected by HsfB1 suppression, but a larger number is differentially abundant in B1OE (Figure 6a). Several Hsps accumulate upon HsfB1 overexpression in control leaves including the stress-induced Hsp101 and the cognate Hsp70-2, whereas BIP4, Hsp21.6-ER, and Hsp17.6-Cll were increased in stems of B1OE plants (Figure 6b; Tables S1 and S2).

Comparing the heat-treated samples yielded a similar number of proteins affected in B1OE and B1AS and generally enhanced number when compared with the control sample (Figure 6a, right). Remarkably, we found several proteins either higher or lower abundant in both, $\mathrm{B} 1 \mathrm{AS}$ and B1OE when compared with WT, but no proteins with contrasting behaviour (e.g., higher in B1OE, lower in B1AS).

Many chaperones showed enhanced levels in HR leaves of B1OE and B1AS plants. We also observed increased levels for the cognates Hsp90-1 and Hsp90-2 in HS samples (Figure 6b). This can be related to the increased thermotolerance. Beyond Hsp70-4 and BIP1, we also identified the Hsp70-Hsp90 interacting co-chaperone HOP and other proteins with housekeeping functions, such as translation elongation 


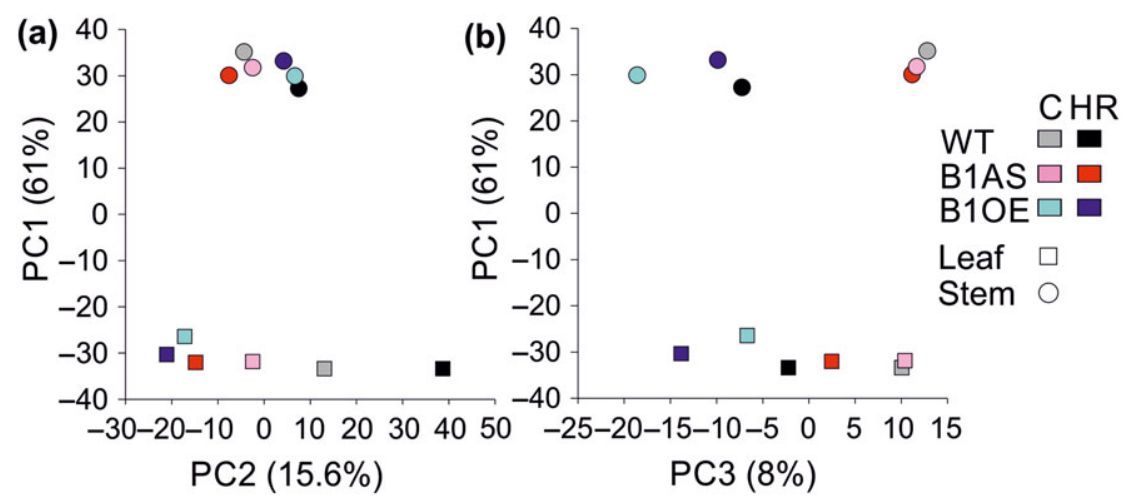

(c)

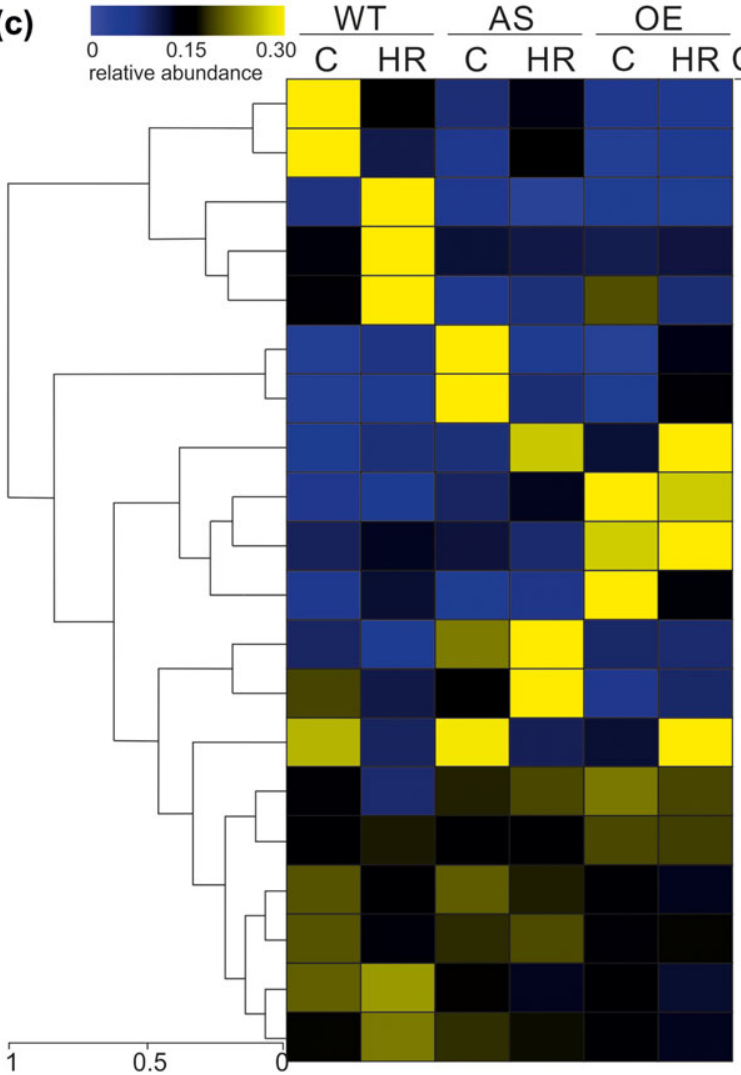

$\begin{array}{lc}\text { 1L } & 40 \\ 1 S & 21 \\ 4 \mathrm{~L} & 33 \\ 5 \mathrm{~L} & 160 \\ 5 \mathrm{~S} & 36 \\ \text { 2L } & 19 \\ 2 \mathrm{~S} & 14 \\ 7 \mathrm{~L} & 61 \\ 3 \mathrm{~L} & 108 \\ 3 \mathrm{~S} & 75 \\ 7 \mathrm{~S} & 19 \\ 6 \mathrm{~L} & 39 \\ 6 \mathrm{~S} & 53 \\ 4 \mathrm{~S} & 64 \\ 8 \mathrm{~L} & 328 \\ 8 \mathrm{~S} & 666 \\ 9 \mathrm{~L} & 442 \\ 9 \mathrm{~S} & 467 \\ 10 \mathrm{~L} & 482 \\ 10 \mathrm{~S} & 297\end{array}$

FIGURE 5 Proteome changes in WT, B1AS, and B1OE leaves and stem from control and heat stressed plants at recovery stage (HR). Principal component analysis was performed and variations of (a) PC1 versus $P C 2$, and (b) PC1 versus PC3 for all examined samples are shown. (c) Protein abundance profile clustering. A hierarchical cluster analysis was performed as described in methods. The average normalized levels of the proteins in leaves (L) and stems (S) of WT and transgenic lines in each cluster are presented in a heat map. The number of spots assigned to each cluster is shown on the right factors (e.g., ID14507/Solyc08g079180), and actin (e.g., ID14836/ Solyc03g078400) that were reduced by HS in WT tissues but sustained steady levels in the transgenic lines (Figure 6b; Dataset S2).

\section{6 | Biological processes related to HsfB1 expression level manipulation}

2D-DIGE allowed the identification of quantitative changes in the abundance of individual proteins; however, due to the low number of spots assigned to single proteins, we were not able to conclude on global changes regarding biological processes affected by HsfB1 manipulation. Thus, we performed a shotgun proteomic analysis by LC-MS/MS on samples derived from plants treated the same way as for 2D-DIGE; 1270 different proteins were detected (Dataset S3) and quantified by MaxQuant for comparative analysis between transgenic lines and WT leaf and stem for each experimental condition, as shown for 2D-DIGE (Figure 5a).
Proteins affected by HsfB1 overexpression and suppression most frequently are involved in translation, abiotic stress response, redox regulation, photosynthesis, and protein folding and degradation in both leaves and stems under control and stress conditions (Figure 7; Dataset S4). Remarkably, proteins of these categories were both increased or reduced in B1AS and B1OE tissues compared with WT. However, a higher number of proteins related to translation and protein degradation was reduced in B1AS and B1OE leaves and stems after heat treatment. Furthermore, most proteins affected by HsfB1 overexpression and suppression in leaves are related to transcription and signalling, whereas in stressed stem tissues, most of the proteins are involved in lipid metabolism as well as cell wall synthesis and modification (Dataset S4).

\subsection{Regulation of transcript abundance by HsfB1}

We selected seven proteins induced or reduced in B1AS or B1OE leaves to analyze whether the differences on protein level 
(a)

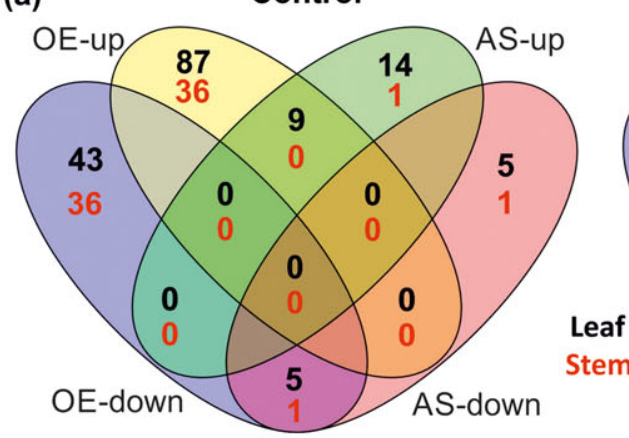

Heat stress - Recovery

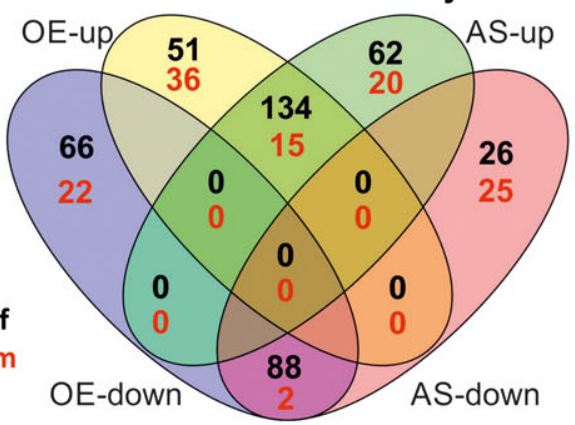

Fold change AS or OE $/ \mathrm{WT}>2$ or $<0.5, p<0.01$

(b)
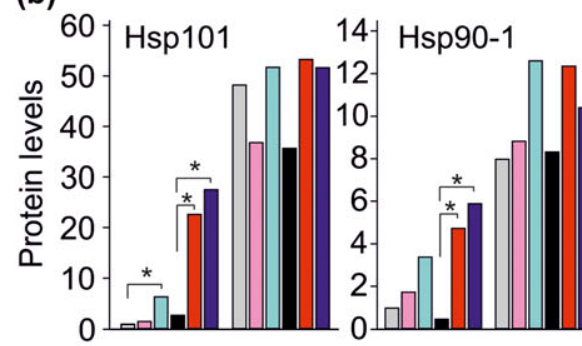

Hsp90-2

Hsp90-5
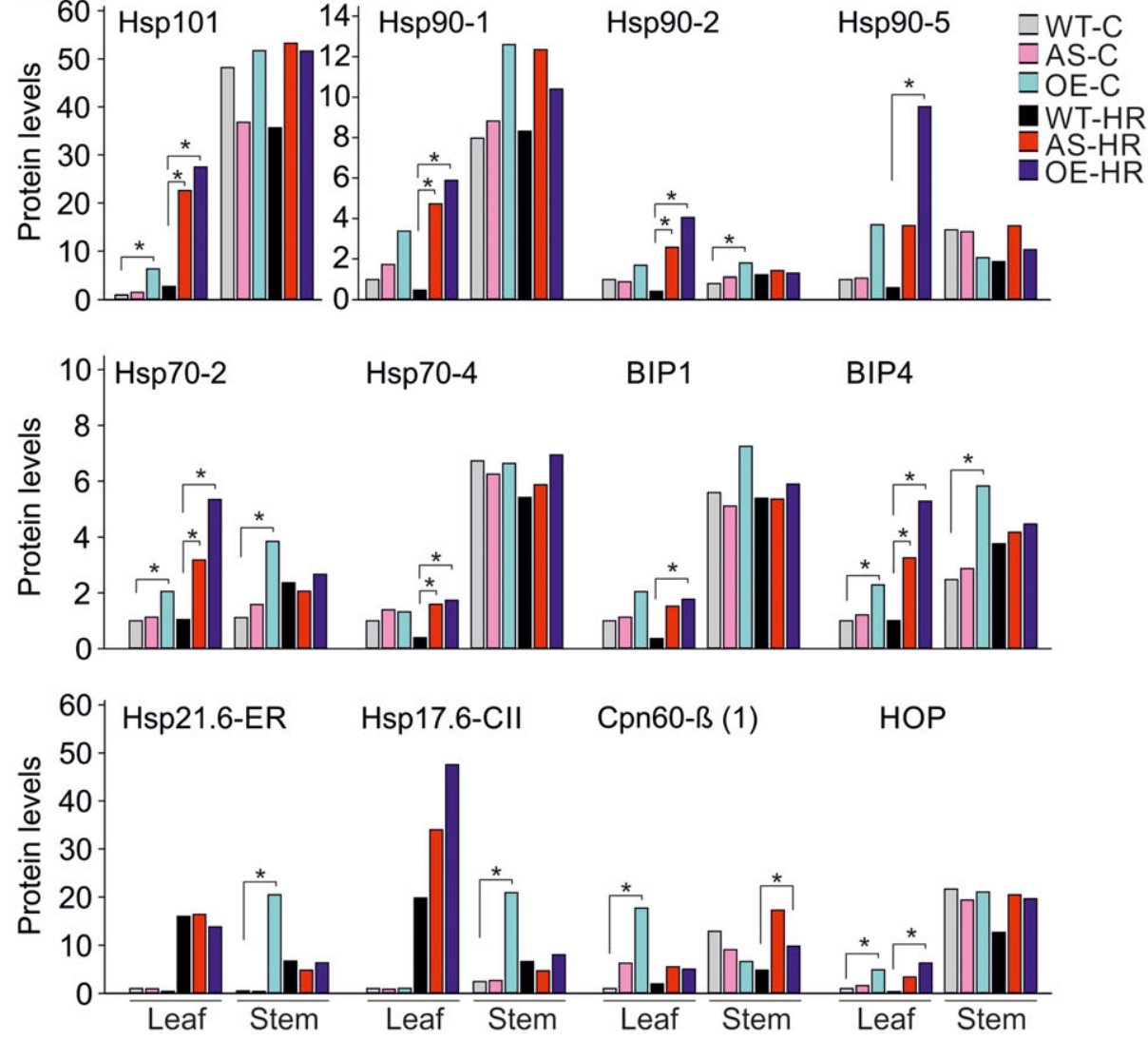

FIGURE 6 Protein affected by HsfB1 suppression or overexpression in leaves and stems. (a) Venn diagram showing the number of identified spots with higher (AS or OE to WT ratio $>2, p<0.01$ ) or reduced levels (AS or OE to WT ratio $<0.5$-fold, $p<0.01$ ) in control and HR samples of leaves and stems (for details, see Dataset S1). (b) Levels of indicated proteins determined by 2D-DIGE showing enhanced or reduced abundance due to HsfB1 overexpression or suppression in control and HR leaves and stems. Asterisks indicate significant differences between the indicated samples (ratio $>2$ or $<0.5, p<0.01$ ) based on the Student's $t$ test

correspond to alterations on the transcript level. We examined the abundance of the according mRNAs isolated from two suppression (AS-18, AS-21) and two overexpression (OE-5, OE41) lines as well as WT by qRT-PCR. The transcript level of none of the genes was affected by HsfB1 suppression or overexpression, with the exception of a gene coding for a putative ATP-dependent chromatin-remodelling protein SWIB/MDM2 (Solyc01g107330). This gene was slightly higher in stressed and non-stressed B1AS lines, and in non-stressed B1OE lines when compared with WT (Figure S4). In line, the putative SWIB/MDM2 protein was more abundant in both AS and OE heat stressed leaves (Figure S4). Thus, we assume that the differences on the levels of most of the proteins observed by 2D-DIGE and LC-MS are not directly correlated to alterations of transcript levels as consequence of HsfB1 expression level manipulation.

As HsfB1 is primarily expected to regulate chaperones, the transcript levels of several Hsps were monitored in $\mathrm{AS}$ and $\mathrm{OE}$ leaves. As the transcriptional activation of stress-induced Hsps occurs during the stress treatment, we included in this analysis an additional sample directly taken after $1 \mathrm{hr}$ of HS. The levels of Hsp101, the cytosolic Hsp90-1, Hsp90-2, Hsp70-1, and Hsp70-3 were comparable in all lines under normal conditions or in the recovery phase (Figure 8). Only after $\mathrm{HS}$, a higher accumulation was observed for these genes in B1AS and B1OE lines when compared with WT, with the exception of Hsp70-3. For Hsp101, the 

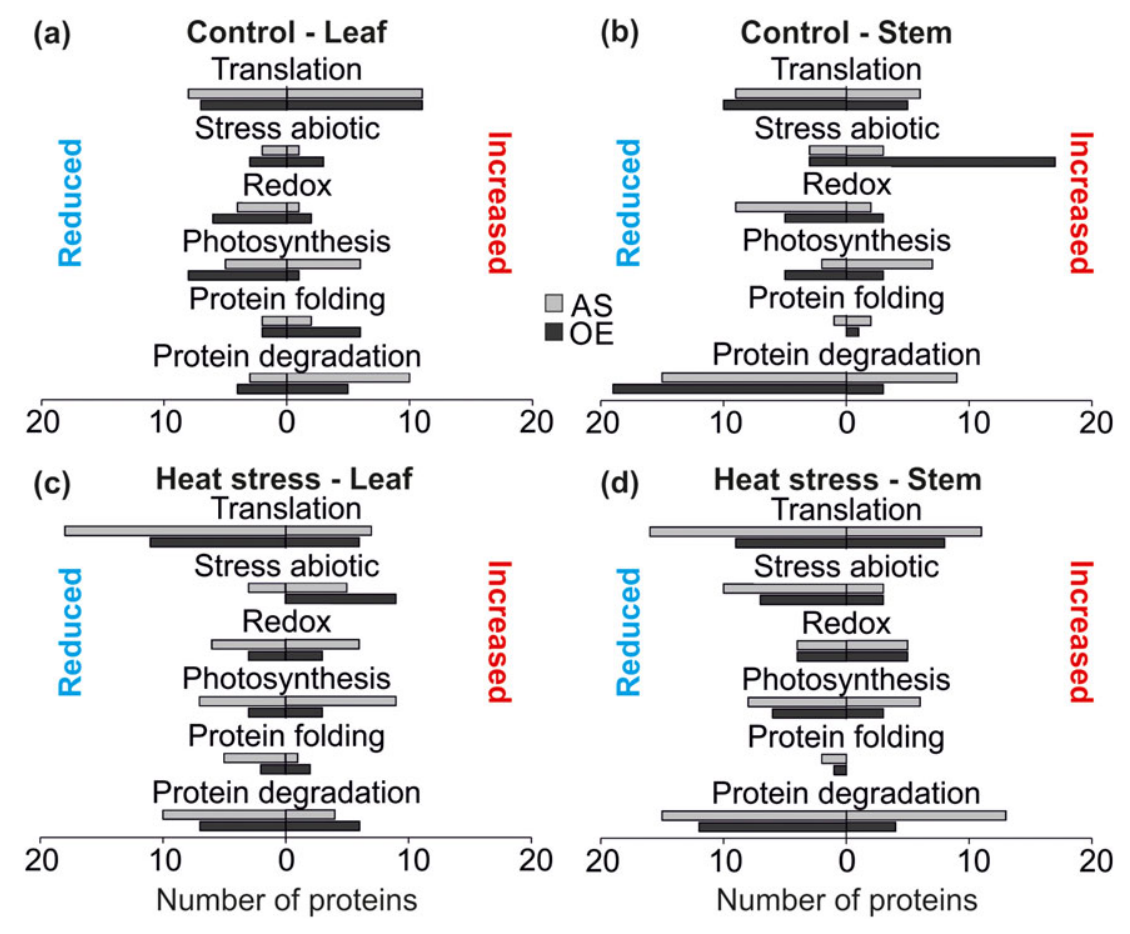

FIGURE 7 Functional categorization of proteins affected by HsfB1 overexpression or suppression in control leaf (a) and stem (b) and HS leaf (c) and stem (d) samples. Protein groups identified by LC-MS/MS analysis were subjected to Mapman functional classification. The categories with the highest number of proteins are shown here, whereas the whole dataset is present in Dataset S4
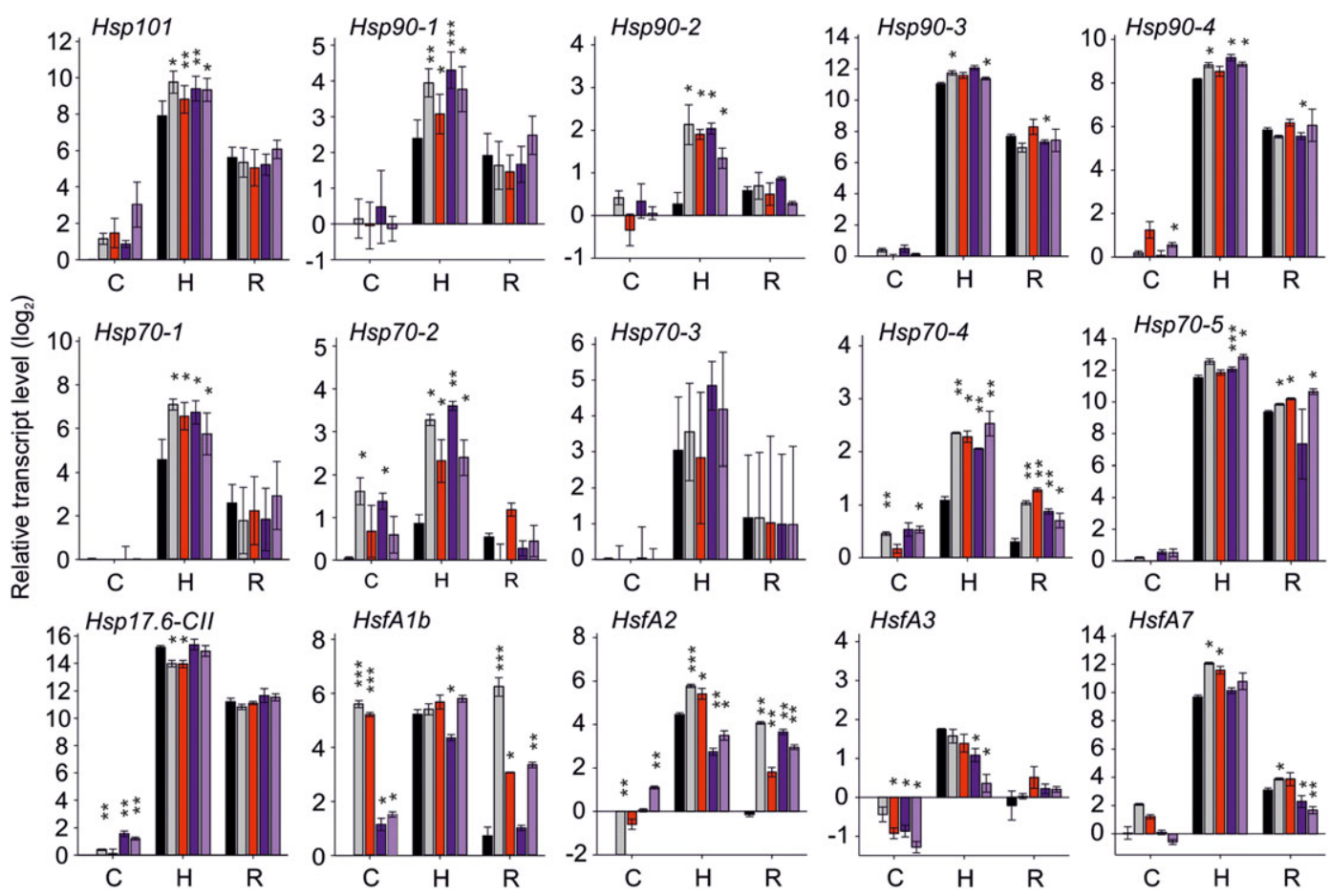

FIGURE 8 Effect of HsfB1 suppression or overexpression on the transcript levels of Hsfs and Hsps. Relative expression of Hsfs and Hsps in leaves of wild-type (WT) antisense (AS-18, AS-21) or sense overexpression lines (OE-5, OE-41) exposed to $39^{\circ} \mathrm{C}$ for $1 \mathrm{hr}(\mathrm{HS})$ followed by recovery (HR) at $25^{\circ} \mathrm{C}$ for $1.5 \mathrm{hr}$ or kept for the entire period at $25^{\circ} \mathrm{C}(\mathrm{C})$ was determined by qRT-PCR using EF1a as housekeeping gene and normalization relative to WT control sample. The average derives from three independent experiments. Statistically significant differences between the transgenic lines and the WT for each condition are denoted by asterisks based on pairwise comparisons with Student's $t$ test $\left({ }^{*} p<0.05,{ }^{* *} p<0.01,{ }^{* * *} p<0.001\right)$

increased accumulation in both $\mathrm{AS}$ and $\mathrm{OE}$ lines after $1.5 \mathrm{hr}$ recovery from stress was confirmed at the protein level as well by immunoblot analysis (Figure S4b).

The stress-induced Hsp90-3 was by large not affected by HsfB1 expression level manipulation under all conditions tested, whereas
Hsp90-4 and Hsp70-5 were more abundant in both OE lines after 1-hr HS. Interestingly, the transcript levels of the Hsp70-2 were increased in heat stressed leaves of B1OE and B1AS plants (Figure 8). Hsp70-4 had enhanced levels after HS and recovery of B1OE and B1AS leaves. Thus, Hsp70 appears to be the gene family 
largely affected in its transcription in the transgenic lines (Figure 8). In contrast, Hsp17.6-CII transcripts were reduced in B1AS heat stressed leaves but were similar to WT in B1OE samples. A slight induction of Hsp17.6-Cll transcripts was observed under control conditions in B1OE leaves.

The major HS-inducible Hsfs were affected in a different manner by HsfB1 suppression and overexpression than Hsps. HsfA1b showed enhanced transcript levels under control conditions in leaves of both B1AS and B1OE lines, with more prominent upregulation in B1AS (Figure 8). After HS, the level of HsfA1b was similar to WT but remained higher in the B1AS plants after recovery. HsfB1 suppression led to an increased accumulation of HsfA2, whereas overexpression of HsfB1 led to reduced induction of HsfA2. After $1.5 \mathrm{hr}$ of recovery, both B1AS and $\mathrm{B} 1 \mathrm{OE}$ plants maintained higher HsfA2 levels. HsfA7 levels were also higher than WT in B1AS heat stressed leaves and were higher in the recovery sample of B1OE leaves when compared with the WT. In contrast, we only noticed a repression of HsfA3 by HsfB1 overexpression in control and HS leaves (Figure 8).

\subsection{Co-activator and repressor activity of HsfB1}

The effect of HsfB1 expression on HS-inducible Hsf and Hsp promoters including the one of HsfA1b and HsfA2 found to be altered in expression in transgenic plants (Figure 8) was examined in a reporter assay. In parallel, the co-activation of HsfA1a by HsfB1 and HsfA2 was tested. HsfA1a has strong activity on HsfA2 and Hsp21.5-ER promoters and weak activity on HsfA1b, HsfA7, Hsp70-1, and Hsp17-Cl (Figures 9a and S5a). HsfB1 repressed the activity of HsfA1b, HsfA2, HsfA7, Hsp70-1, and Hsp21.5-ER, but had no significant effect on Hsp17*-Cl (Figure 9a). Co-expression of HsfA1a and HsfB1 led to a stronger induction of GUS activity on $\mathrm{Hsp} 17^{*}-\mathrm{Cl}$ and a slighter increase in Hsp70-1 when compared with HsfA1a alone. In contrast, co-expression of HsfB1 with HsfA1a represses the transactivation activity of HsfA1a on the three Hsf promoters, whereas it did not significantly affect HsfA1a activity on the Hsp21.5-ER promoter (Figure $9 a$ and $\mathrm{S} 5 \mathrm{a}$ ). In A. thaliana, HsfA1b shares the master regulator function with the other HsfA1 genes. As HsfA1a and HsfB1 form a coactivator complex on the $\mathrm{Hsp} 17$ promoter (Figure 9a), we examined whether HsfB1 co-activates Hsp17 expression driven by other class A Hsfs. HsfA1b has only minor activity per se and does not act as co-activator of HsfA1a or HsfB1 (Figure S6). However, HsfA1b has synergistic activity with $\mathrm{HsfA} 2$ and $\mathrm{HsfA}$, which are both HS-induced proteins. These results suggest that the accumulation of HsfA1b in AS plants under control conditions has no effect on regulation of HS-induced genes, but its presence can cause a stronger induction of HS-related genes under stress conditions when HsfA2 and HsfA3 are present (Figure $\mathrm{S6}$ ).

HsfB1 acts as a co-activator or repressor of HsfA1a in a promoter-dependent manner (Figure 9a). To examine whether this is restricted to a co-action with $\mathrm{HsfA} 1 \mathrm{a}$, we co-expressed in protoplasts combinations of HsfB1 with several class A Hsfs and determined their activity on HsfA2 and $\mathrm{Hsp} 17^{*}-\mathrm{Cl}$ promoters using the GUS reporter assay (Figures $9 \mathrm{~b}$ and $\mathrm{S} 5 \mathrm{~b}$ ). In addition to HsfA1a shown before, HsfA1b, HsfA3, HsfA6b, HsfA7, and HsfA9 had a basal activity on the HsfA2 promoter, whereas HsfA4b alone had a very strong activation potential. In all cases, co-expression of HsfB1 had a clear repressor effect with all Hsfs examined.

The picture was different when the Hsp17 promoter was tested. Here, HsfB1 acts as a co-activator only with HsfA1a, HsfA2, HsfA6b, and HsfA9; however, the synergistic activity is the strongest for the HsfA1a-HsfB1 co-activator complex (Figures 9b and S5b). In contrast, in presence of HsfB1, HsfA4b and HsfA7 were repressed irrespective of the promoter tested.

The stimulatory effect of HsfA1a-HsfB1 is much stronger, leading to a 13-fold induction in GUS activity, compared with the activity of HsfA1a alone. In turn, the activity of HsfB1 with HsfA2, HsfA6b, and HsfA9 ranges from 0.3-fold to sixfold (Figure 9b). We asked if the weak activation conferred by HsfB1 co-expression with some Hsfs, like HsfA2 is dependent on the endogenous HsfA1a. For this, the activity of Hsf-dependent PHsp17*::GUS reporter construct was determined in the presence of constant levels of HsfA2 and increasing amounts of HsfB1 in WT protoplasts (Figure 9c). HsfB1 stimulated the activity of HsfA2 in WT protoplasts. In contrast, HsfB1 failed to co-activate HsfA2 when both proteins were co-expressed in A1CS protoplasts derived from transgenic plants with HsfA1a co-suppression (Mishra et al., 2002; Figure 9c). Similar results were obtained in WT and A1CS protoplasts expressing HsfB1 and HsfA3 (Figure S7). These results suggest that HsfB1 can modulate the activity of the Hsf network in an HsfA1a-dependent manner.

\section{4 | DISCUSSION}

\subsection{Effects of HsfB1 in non-stressed cells}

The functional relevance of HsfB1 for development, growth, and thermotolerance was examined using three independent HsfB1 overexpression and suppression plant lines, respectively. Phenotypic, gene expression, and proteome analyses revealed that overexpression and suppression of HsfB1 yield unique as well as similar responses. The latter highlights the functional complexity of HsfB1 in tomato due to its seemingly unique ability among Hsfs to act as repressor as well as co-activator (Bharti et al., 2004). Extending the initial evidence on the dual function of HsfB1 based on transient and reporter assays (Bharti et al., 2004), we provide insights into the regulation of Hsf-dependent networks by HsfB1 and their relation to physiological responses under control and aberrant temperatures.

HsfB1 protein is kept at minimal levels under non-stress conditions (Figure 1) by Hsp90-dependent protein degradation (Hahn et al., 2011; Röth et al., 2016). Nevertheless, suppression of HsfB1 leads to changes of the abundance of several transcripts (Figure 8) and proteins under normal growth conditions (Figure 5, clusters $1 \mathrm{~L} / \mathrm{S}$ and $2 \mathrm{~L} / \mathrm{S}$; Figures 6 and 7). Irrespective whether these alterations are direct effects of HsfB1 manipulation or phenotypic effects due to alteration of the Hsf-involving network, these findings document a basal activity of HsfB1 in leaves and stems of non-stressed plants.

Although manipulation of HsfB1 results in alterations of transcript and protein levels (Figures 5-8), B1AS/CS plants show 

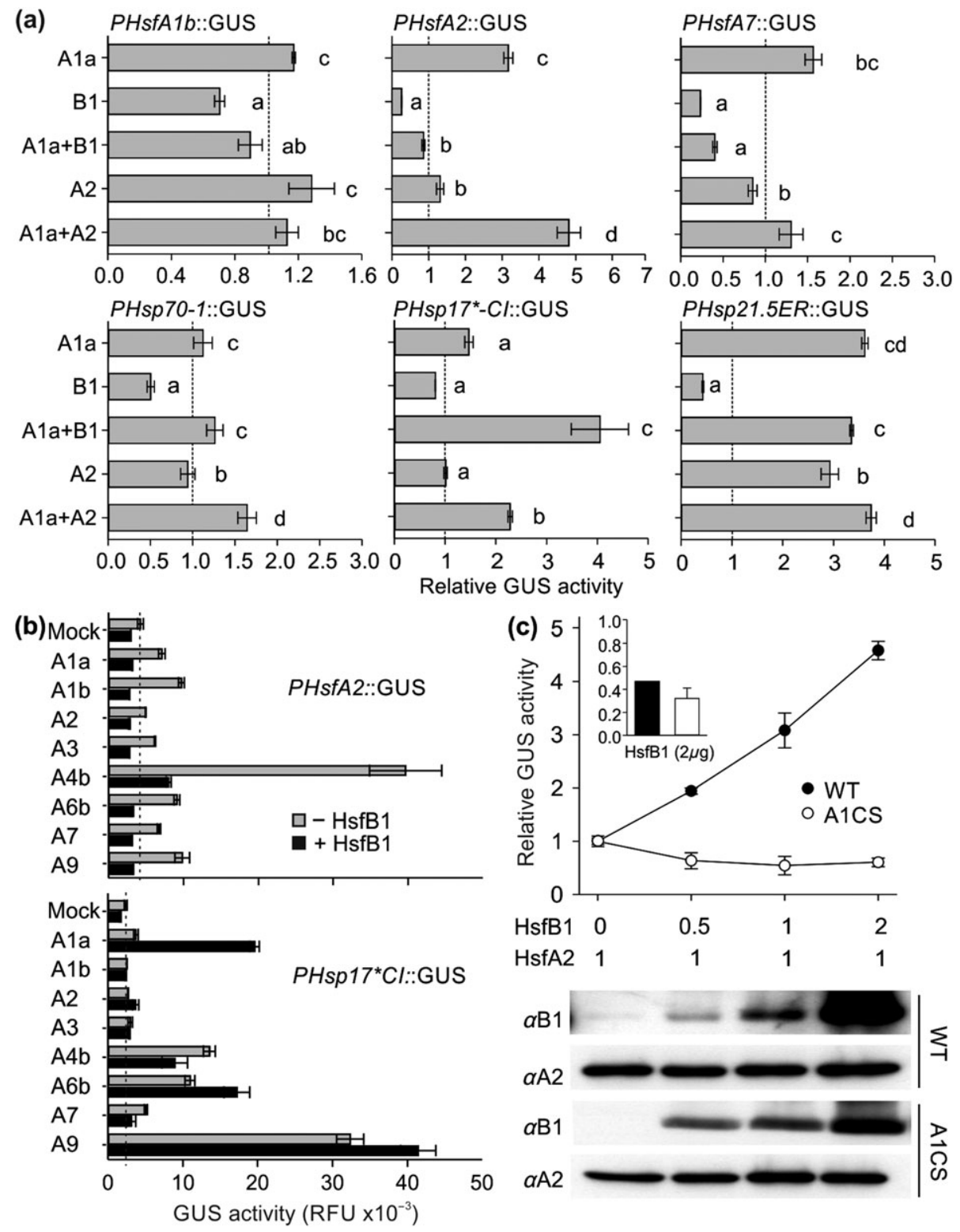

FIGURE 9 Regulation of Hsf and Hsp activity by HsfB1. (a) Tomato protoplasts were co-transformed with different promoter GUS reporter plasmids together with expression constructs encoding HsfA1a, HsfB1, and HsfA2. The values are expressed as relative to a mock control (no Hsf). The red line depicts the level of the mock sample (onefold). Different letters show statistically significant differences as calculated by ANOVA and Duncan's test for $p<0.05$. Error bars show the standard error of three replicates. (b) Activity of HsfB1 in combination with other Hsfs on PHsfA2::GUS and PHsp17*::GUS reporters in wild-type protoplasts. The values are the mean of three replicates of GUS activities in relative fluorescence units (RFU) \pm SE. (c) Tomato protoplasts from wild-type and A1CS leaves were co-transformed with the PHsp17*::GUS reporter together with constant amount of HsfA2 expression constructs and increasing amounts of HsfB1 indicated as microgram of plasmid DNA. GUS activities are represented as relative to the activity in protoplasts transformed with only HsfA2 for each genotype. The insert shows the repressor activity of HsfB1 on the sHsp promoter in WT and A1CS protoplasts. The values are the mean of three independent replicates \pm SD. The protein levels of HsfB1 and HsfA2 are shown on the immunoblots using specific antibodies against the two proteins

the same phenotype as WT (Figure 2), and the protein pattern is comparable for B1AS and WT under non-stress conditions (Figure 5, leaf, PC2). A similar result was observed for A. thaliana single $h s f b 1$ and double knock-out hsfb1-hsfb2b mutants that were phenotypically similar to WT under control conditions with the exception of elongated hypocotyls in young seedlings (Ikeda et al., 2011; Kumar et al., 2009). We only detected a lower photosynthetic rate in old leaves of the antisense line (Figure 4) and a slightly stronger apical dominance and longer basal internodes, which did not affect the overall stem length (Figure 2).
We conclude that HsfB1 is not essential for tomato plant growth and development, but its basal activity is required for optimum, eg, of photosynthetic performance and for maintaining a low activity of Hsfs, such as HsfA1b and consequently of several Hsps under non-stress conditions. In contrast to the effect of HsfB1 suppression in tomato, $A$. thaliana $h s f b 1-h s f b 2 b$ mutants are characterized by a dramatic upregulation of HS-induced genes including several Hsfs and sHsps (Ikeda et al., 2011), which is not apparent in tomato B1AS lines. This marks a major difference between the two Hsf systems. 


\subsection{HsfB1 links environmental signals to developmental and growth responses}

Ectopic expression of HsfB1 protein accumulates in the overexpression lines under non-stress conditions (Figure 1c). The ectopic levels of HsfB1 lead to reduced stem growth, weaker apical dominance, and lower fruit set (Figure 2). B1OE leaves show increased photosynthetic rate and a higher NPQ indicating an induction of protective mechanisms for photosystem II under non-stress conditions (Figure 4). The thylakoid-bound ascorbate peroxidase 6 (tAPX6, Solyc11g018550; LC-MS/MS) and the plastidic chaperonin Cpn60- $\beta$ (Solyc01g028810; 2-DIGE and LC-MS/MS) are enhanced in B1OE leaves (Tables S2 and S4). In line, mutants of wheat TaAPX-6B show lower photosystem II ETR and photosynthetic activity under high-intensity light conditions (Danna et al., 2003), whereas plastid chaperonins are important for the biogenesis of RuBisCO (Vitlin, Nisemblat, Azem, \& Weiss, 2013). Supporting the notion that enhanced chaperonin levels could lead to increased biogenesis of RuBisCO, spots corresponding to the large subunit of RuBisCO were identified in cluster $3 \mathrm{~L}$, containing proteins with higher levels in $\mathrm{OE}$ leaves (Dataset S2). We assume that the accumulation of these proteins contributes to the increased photosynthetic rate in B1OE leaves.

The phenotype of B1OE-reduced growth, alterations in apical dominance, and reduced fruit set-resembles the one of heat stressed plants (Sato, Peet, \& Thomas, 2000), which is in line with the low separation between control and HS B1OE samples in PCA (Figure 5a,b). In this direction, several Hsps are enhanced in $\mathrm{OE}$ leaves and stems (Figure 6; Tables S1-S4). The latter likely depends on the cooperation of HsfB1 with HsfA1a through the formation of ternary complexes with HAC1 in stressed cells (Bharti et al., 2004). Thus, the ectopic accumulation of HsfB1 enhances the basal activity of HsfA1a on Hsp activation. The activation of HsfAla by HsfB1 accumulation is also supported by the lost of co-activator capacity of HsfB1 on HsfA2 and HsfA3 in A1CS protoplasts (Figures 9c and S7). One explanation might be that the accumulation of HsfB1 reliefs HsfA1a from chaperone-mediated repression by enhancing its recruitment in ternary activator complexes (Bharti et al., 2004; Hahn et al., 2011).

The upregulation of cognate Hsps, such as Hsp70-2, which are not regulated by class A Hsfs (Fragkostefanakis et al., 2015, 2016), indicates a co-activator function of HsfB1 for non-Hsf transcription factors as previously suggested (Bharti et al., 2004). The accumulation of Hsps under non-stress conditions in $\mathrm{B} 1 \mathrm{OE}$ lines is also consistent with the growth and developmental defects, as overexpression of HSC70-1 in A. thaliana caused similar phenotypes (Cazalé et al., 2009). Such an accumulation of cognate Hsps was not observed in HsfA1a overexpression plants (Mishra et al., 2002), which can explain the absence of developmental and growth defects of A1OE transgenic plants.

In line, we detected higher steady-state levels of several proteins in B1OE leaves involved in translation processes such as tRNA synthetases, ribosomal proteins, elongation factors, and several eukaryotic translation initiation factors (Figure 6; Tables S1 and S4). The higher levels of such proteins might support a more robust protein translation under stress conditions and subsequently explain the increased protein levels of several proteins that are not regulated by HsfB1 at the transcript level (Figure S4a). Analysis of the thermosensitive hot3-1 mutant in A. thaliana revealed the essential role of elF5B in initiation of translation (Zhang et al., 2017). In addition the higher accumulation of chaperones can also promote protection and stability of translation factors, as, for example, $\mathrm{Cl}$ and $\mathrm{Cll}$ sHsps are involved in the recovery of translation factors from stress granules during recovery from stress, in a process that involves Hsp101 as well (McLoughlin et al., 2016). These results point to a role of HsfB1 as regulator of cellular homeostasis networks via modulation of chaperone networks.

\section{3 | Co-activation and repression by HsfB1 fine tune heat stress response and thermotolerance}

HsfB1 expression level manipulation led to increased capacity of seedlings to recover from HS by enhancing both BTT and ATT (Figure 3). Moreover, B1OE and B1AS old leaves showed increased NPQ indicating higher protection of photosystems despite the differences in photosynthetic rates (Figure 4). In B1OE, the increased thermotolerance might result from the accumulation of proteins with protective functions under control conditions or from a stronger induction or maintenance of higher protein levels in HS samples (e.g., Hsp90-1, Hsp90-2; Figures $6 \mathrm{~b}$ and 8 ). The increased accumulation of chaperones in stressed B1OE leaves can be explained by the enhanced levels of Hsps already induced under control conditions due to the co-activator function of HsfB1 (Figures 8, 9, and S4b). In turn, suppression of HsfB1 did not have significant effects under control conditions when compared with WT, but allowed a stronger accumulation of chaperones in response to HS, leading to enhanced protection (Figures $6 \mathrm{~b}, 8$, and S4b).

In contrast to the currently available model for HsfB1 (Mishra et al., 2002), our results indicate that the co-activator function of HsfB1 with HsfA1a is promoter specific. HsfB1 acts as co-activator on promoters of Hsps and as a repressor to HsfA1a on promoters of HsfA1b, HsfA2 and HsfA7 (Figure 9). Consequently, these three Hsfs show enhanced transcript levels in either control or in stressed B1AS samples, and therefore, the transactivation reporter assays are in agreement with the responses of the transgenic plants. This is in line with the stronger stress response and protection in the absence of HsfB1 (Figures 3-5 and 8). Remarkably, the repressor function of HsfB1 on these promoters is not rescued by any of the examined Hsfs, and even the highly active HsfA4b on the HsfA2 promoter was largely repressed in the presence of HsfB1 (Figure 9b). We conclude that HsfB1 has a general repressor effect for at least a subset of HS genes linked to the activity of HS-induced Hsfs.

The induction of HsfA1b in B1AS plants did not result in an accumulation of stress-induced genes due to the very weak transcriptional activator capacity of HsfA1b (Figure S6). Instead, a preformed HsfA1b has strong synergistic activity with HsfA2 or HsfA3 under HS, which supports the absence of a phenotype in B1AS plants under control conditions, and explains the stronger response under HS compared with WT. For the latter, the increased accumulation of HsfA2 likely contributes to enhanced stress response and thermotolerance as well. In a similar manner, the A. thaliana hsfb1-hsfb2b mutant showed enhanced HsfA2 levels and accordingly increased levels of several stress induced Hsps (Ikeda et al., 2011; Kumar et al., 2009). 


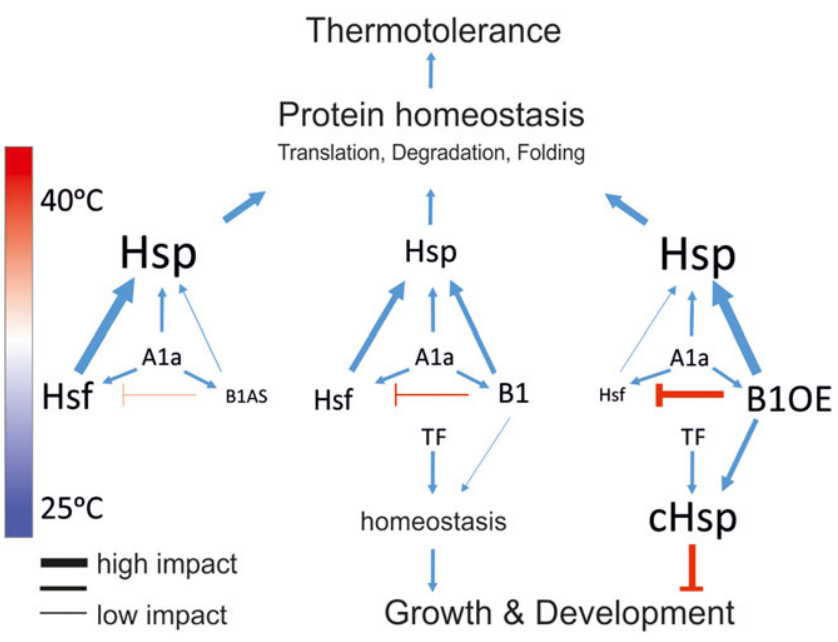

FIGURE 10 Proposed model for the role of the bifunctional HsfB1 in modulation of cellular homeostasis. HsfB1 plays a central role in fine regulation of HSR and thermotolerance capacity. HsfB1 acts as a coactivator of HsfA1a, thereby, accumulation of HsfB1 leads to increased synthesis of chaperones, particularly cognate Hsps, probably as a co-regulator of other transcription factors (TF) even under nonstress conditions as shown in OE lines. The activation of HSR networks under physiological conditions results in aberrant growth and development. In addition, HsfB1 acts as a direct transcriptional repressor of stress induced Hsfs. Consequently, suppression of HsfB1 (AS lines) leads to increased accumulation of Hsfs, including HsfA1b, HsfA2, and HsfA7 and consequently to stronger activation of HSR and synthesis of Hsps. The stronger accumulation of Hsps leads to the induction of proteostasis-related protective mechanisms not directly regulated by HsfB1, to ensure thermotolerance and surveillance. For further details, see text

We could not identify proteins that are enhanced in B1OE and repressed in B1AS, or vice versa, neither in control nor in HS samples (Figure 7a; Dataset S4). This compromises the assignment of an exclusive co-activator or a repressor function of HsfB1 to specific genes, with the exception of HsfA2 under HS conditions (Figure 8). The latter explains the similar effect of HsfB1 overexpression and suppression on many Hsps under HS, as overexpression stimulates transcription due to the co-activator function of HsfB1, whereas the same occurs when HsfB1 is suppressed due to the relief of its repressor function on other Hsfs (Figure 9). In line, many proteins with similar biological functions were affected in the same manner by HsfB1 overexpression or suppression (Figure 7; Dataset S4). Thus, HsfB1 is a central component of a versatile regulatory system and not by itself target specific. The regulatory system ensures protection from HS by the fine adjustment of protein homeostasis, for example, by enhancing chaperone capacity (Figures 6 and 8) or reducing translation and protein degradation (Figure 7). All of them ensure proteome stability (Figure S3) and eventually increase thermotolerance (Figures 3 and 4).

\section{5 | CONCLUSIONS}

We propose that HsfB1 fine tunes the regulatory network by coactivation of HsfA1a activity on HS-inducible genes and repression of Hsf expression, for example, of HsfA1b and HsfA2 (Figure 10).
Consequently, the increase and the decrease of the homeostatic HsfB1 levels activate far-reaching responses through induction of Hsps by either direct activation or by reduction of Hsf repression. The response occurs by triggering forward and feedback reactions through chaperone-controlled networks similar to those induced by changes in ambient temperatures. This marks HsfB1 as central control unit of complex networks that interfere with the regulation of multitude processes involved in adaptation and maintenance of the cellular homeostasis under constantly changing environmental conditions (Figure 10). At least in part, this would explain the strict requirement for the tight control of HsfB1 levels in non-stressed and stressed plants (Hahn et al., 2011; Röth et al., 2016) to ensure sufficient robustness for optimal plant growth and development.

\section{ACKNOWLEDGEMENTS}

We thank Holger Schranz for maintenance of the plants in the greenhouse, Julia Sachs, Matthias Reiter, and Sascha Röth for technical assistance and critical comments during the preparation of the manuscript, and Lutz Nover for long-term endorsement and critical attendance of the work. We would like to thank for financial support from Bundesministerium für Bildung und Forschung to K.D.S., China Scholarship Council to Y.H., Deutsche Forschungsgemeinschaft (SFB902) to E.S., Deutscher Akademischer Austausch Dienst Kairo to A.S., and Marie Curie Initial Training Network SPOT-ITN to E.S. and K.D.S.

\section{CONFLICT OF INTEREST}

All authors have read and approved the manuscript. Authors declare that they have no conflict of interest.

\section{ORCID}

Shravan Kumar Mishra (D) http://orcid.org/0000-0003-3899-0495

Enrico Schleiff (DD http://orcid.org/0000-0002-0518-3489

\section{REFERENCES}

Baniwal, S. K., Kwan, Y. C., Scharf, K. D., \& Nover, L. (2007). Role of heat stress transcription factor HsfA5 as specific repressor of HsfA4. Journal of Biological Chemistry, 282, 3605-3613.

Becker, D., Kemper, E., Schell, J., Masterson, R., Ziichtungsforschung, M., Weg, L., \& Koln, D. (1992) New plant binary vectors with selectable markers located proximal to the left T-DNA border. 1195-1197.

Bharti, K., Schmidt, E., Lyck, R., Heerklotz, D., Bublak, D., \& Scharf, K. D. (2000). Isolation and characterization of HsfA3, a new heat stress transcription factor of Lycopersicon peruvianum. Plant Journal, 22, 355-365.

Bharti, K., Von Koskull-Döring, P., Bharti, S., Kumar, P., Tintschl-Körbitzer, A., Treuter, E., \& Nover, L. (2004). Tomato heat stress transcription factor HsfB1 represents a novel type of general transcription coactivator with a histone-like motif interacting with the plant CREB binding protein ortholog HAC1. The Plant Cell, 16, 1521-1535.

Bokszczanin, K. L., \& Fragkostefanakis, S. (2013). Perspectives on deciphering mechanisms underlying plant heat stress response and thermotolerance. Frontiers in Plant Science, 4, 315.

Bradford, M. M. (1976). A rapid and sensitive method for the quantitation of microgram quantities of protein utilizing the principle of protein-dye binding. Analytical Biochemistry, 72, 248-254.

Cazalé, A. C., Clément, M., Chiarenza, S., Roncato, M. A., Pochon, N., Creff, A., ... Noël, L. D. (2009). Altered expression of cytosolic/nuclear HSC70-1 molecular chaperone affects development and abiotic stress 
tolerance in Arabidopsis thaliana. Journal of Experimental Botany, 60 2653-2664.

Chan-Schaminet, K. Y., Baniwal, S. K., Bublak, D., Nover, L., \& Scharf, K.-D. (2009). Specific interaction between tomato HsfA1 and HsfA2 creates hetero-oligomeric superactivator complexes for synergistic activation of heat stress gene expression. The Journal of Biological Chemistry, 284, 20848-20857.

Charng, Y. Y., Liu, H. C., Liu, N. Y., Chi, W. T., Wang, C. N., Chang, S. H., \& Wang, T. T. (2006). A heat-inducible transcription factor, HsfA2, is required for extension of acquired thermotolerance in Arabidopsis. Plant Physiology, 143, 251-262.

Czarnecka-Verner, E., Pan, S., Salem, T., \& Gurley, W. B. (2004). Plant class B HSFs inhibit transcription and exhibit affinity for TFIIB and TBP. Plant Molecular Biology, 56, 57-75.

Danna, C. H., Bartoli, C. G., Sacco, F., Ingala, L. R., Santa-María, G. E., Guiamet, J. J., \& Ugalde, R. A. (2003). Thylakoid-bound ascorbate peroxidase mutant exhibits impaired electron transport and photosynthetic activity. Plant Physiology, 132, 2116-2125.

Döring, P., Treuter, E., Kistner, C., Lyck, R., Chen, A., \& Nover, L. (2000). The role of AHA motifs in the activator function of tomato heat stress transcription factors HsfA1 and HsfA2. The Plant Cell, 12, 265-278.

Eymann, C., Homuth, G., Scharf, C. \& Hecker, M. (2002). Bacillus subtilis functional genomics: Global characterization of the stringent response by proteome and transcriptome analysis. Journal of Bacteriology. 184, $2500-2520$

Fragkostefanakis, S., Mesihovic, A., Simm, S., Paupière, M. J., Hu, Y., Paul, P., ... Scharf, K.-D. (2016). HsfA2 controls the activity of developmentally and stress-regulated heat stress protection mechanisms in tomato male reproductive tissues. Plant Physiology, 170, 2461-2477.

Fragkostefanakis, S., Simm, S., Paul, P., Bublak, D., Scharf, K. D., \& Schleiff, E. (2015). Chaperone network composition in Solanum lycopersicum explored by transcriptome profiling and microarray meta-analysis. Plant, Cell and Environment, 38, 693-709.

Fragkostefanakis, S., Simm, S., Paul, P., Bublak, D., Scharf, K.-D., \& Schleiff, E. (2014). Chaperone network composition in Solanum lycopersicum explored by transcriptome profiling and microarray meta-analysis. Plant, Cell \& Environment.

Giorno, F., Wolters-Arts, M., Grillo, S., Scharf, K. D., Vriezen, W. H., \& Mariani, C. (2010). Developmental and heat stress-regulated expression of HsfA2 and small heat shock proteins in tomato anthers. Journal of Experimental Botany, 61, 453-462.

Hahn, A., Bublak, D., Schleiff, E., \& Scharf, K.-D. (2011). Crosstalk between $\mathrm{Hsp} 90$ and $\mathrm{Hsp} 70$ chaperones and heat stress transcription factors in tomato. The Plant Cell, 23, 741-755.

Hammer, E., Phong, T. Q., Steil, L., Klingel, K., Salazar, M. G., Bernhardt, J., ... Völker, U. (2010). Viral myocarditis induced by Coxsackievirus B3 in A.BY/SnJ mice: Analysis of changes in the myocardial proteome. Proteomics, 10, 1802-1818.

Howe, E. A., Sinha, R., Schlauch, D., \& Quackenbush, J. (2011). RNA-Seq analysis in MeV. Bioinformatics, 27, 3209-3210.

Ikeda, M., Mitsuda, N., \& Ohme-Takagi, M. (2011). Arabidopsis HsfB1 and $\mathrm{HsfB} 2 \mathrm{~b}$ act as repressors of the expression of heat-inducible Hsfs but positively regulate the acquired thermotolerance. Plant Physiology, 157, 1243-1254.

Ikeda, M., \& Ohme-Takagi, M. (2009). A novel group of transcriptional repressors in arabidopsis. Plant and Cell Physiology, 50, 970-975.

Keller, A. D., Nesvizhskii, A. I., Kolker, E., \& Aebersold, R. (2002). Empirical statistical model to estimate the accuracy of protein identifications made by MS/MS and database search. Analytical Chemistry, 74, 5383-5392.

Koncz, C., \& Schell, J. (1986). The promoter of TL-DNA gene 5 controls the tissue-specific expression of chimaeric genes carried by a novel type of Agrobacterium binary vector. Molecular and General Genetics MGG, 204, 383-396.
Kumar, M., Busch, W., Birke, H., Kemmerling, B., Nürnberger, T., \& Schöffl, F. (2009). Heat shock factors HsfB1 and HsfB2b are involved in the regulation of Pdf1.2 expression and pathogen resistance in Arabidopsis. Molecular Plant, 2, 152-165.

Lämke, J., Brzezinka, K., Altmann, S., \& Bäurle, I. (2016). A hit-and-run heat shock factor governs sustained histone methylation and transcriptional stress memory. The EMBO Journal, 35, 162-175.

Lavania, D., Dhingra, A., Siddiqui, M. H., Al-Whaibi, M. H., \& Grover, A. (2015). Current status of the production of high temperature tolerant transgenic crops for cultivation in warmer climates. Plant Physiology and Biochemistry, 86, 100-108.

Liu, H. C., Liao, H. T., \& Charng, Y. Y. (2011). The role of class A1 heat shock factors (HSFA1s) in response to heat and other stresses in Arabidopsis. Plant, Cell and Environment, 34, 738-751.

Lendeckel, D., Eymann, C., Emicke, P., Daeschlein, G., Darm, K., O'Neil, S., ... Scharf, C. (2015). Proteomic Changes of Tissue-Tolerable Plasma Treated Airway Epithelial Cells and Their Relation to Wound Healing. BioMed Research International.

Lê, S., Josse, J., \& Husson, F. (2008) FactoMineR: An R Package for Multivariate Analysis. Journal of Statistical Software. 25, 1-18.

Livak, K. J., \& Schmittgen, T. D. (2001). Analysis of relative gene expression data using real-time quantitative PCR and the $2^{-\triangle \triangle C T}$ method. Methods, 25, 402-408.

McLoughlin, F., Basha, E., Fowler, M. E., Kim, M., Bordowitz, J., KatiyarAgarwal, S., \& Vierling, E. (2016). Class I and II small heat-shock proteins protect protein translation factors during heat stress. Plant Physiology, 172, 00536.2016.

Mesihovic, A., lannacone, R., Firon, N., \& Fragkostefanakis, S. (2016). Heat stress regimes for the investigation of pollen thermotolerance in crop plants. Plant Reproduction, 29, 93-105.

Mishra, S. K., Tripp, J., Winkelhaus, S., Tschiersch, B., Theres, K., Nover, L., \& Scharf, K.-D. (2002). In the complex family of heat stress transcription factors, HsfA1 has a unique role as master regulator of thermotolerance in tomato. Genes \& Development, 16, 1555-1567.

Nesvizhskii, A. I., Keller, A., Kolker, E., \& Aebersold, R. (2003). A statistical model for identifying proteins by tandem mass spectrometry. Analytical Chemistry, 75, 4646-4658.

Nishtala, K., Phong, T. Q., Steil, L., Sauter, M., Salazar, M. G., Kandolf, R., ... Hammer, E. (2013). Proteomic analyses of age related changes in A.BY/ SnJ mouse hearts. Proteome Science, 11, 29.

Nover, L., Bharti, K., Döring, P., Mishra, S. K., Ganguli, A., \& Scharf, K. D. (2001). Arabidopsis and the heat stress transcription factor world: how many heat stress transcription factors do we need? Cell Stress \& Chaperones, 6, 177-189.

Ohama, N., Sato, H., Shinozaki, K., \& Yamaguchi-Shinozaki, K. (2016). Transcriptional regulatory network of plant heat stress response. Trends in Plant Science, 22, 53-65.

Palm, D., Simm, S., Darm, K., Weis, B. L., Ruprecht, M., Schleiff, E., \& Scharf, C. (2016). Proteome distribution between nucleoplasm and nucleolus and its relation to ribosome biogenesis in Arabidopsis thaliana. RNA Biology, 13, 441-454.

Port, M., Tripp, J., Zielinski, D., Weber, C., Heerklotz, D., Winkelhaus, S., ... Scharf, K.-D. (2004). Role of Hsp17.4-CII as coregulator and cytoplasmic retention factor of tomato heat stress transcription factor HsfA2. Plant Physiology, 135, 1457-1470.

Qu, A.-L., Ding, Y.-F., Jiang, Q., \& Zhu, C. (2013). Molecular mechanisms of the plant heat stress response. Biochemical and Biophysical Research Communications, 432, 203-207.

Röth, S., Mirus, O., Bublak, D., Scharf, K-D., \& Schleiff, E. (2016). DNAbinding and repressor function are prerequisite for the turnover of the tomato heat stress transcription factor HsfB1. The Plant Journal: For Cell and Molecular Biology, 1-14.

Sato, S., Peet, M. M., \& Thomas, J. F. (2000). Physiological factors limit fruit set of tomato (Lycopersicon esculentum Mill.) under chronic, mild heat stress. Plant, Cell and Environment, 23, 719-726. 
Scharf, K. D., Berberich, T., Ebersberger, I., \& Nover, L. (2012). The plant heat stress transcription factor (Hsf) family: Structure, function and evolution. Biochimica et Biophysica Acta, 1819, 104-119.

Scharf, K. D., Heider, H., Höhfeld, I., Lyck, R., Schmidt, E., Nover, L., ... Nover, L. (1998). The tomato Hsf system: HsfA2 needs interaction with HsfA1 for efficient nuclear import and may be localized in cytoplasmic heat stress granules. Molecular and Cellular Biology, 18, 2240-2251.

Scharf, K. D., Rose, S., Zott, W., Schöff, F., \& Nover, L. (1990). Three tomato genes code for heat stress transcription factors with a region of remarkable homology to the DNA-binding domain of the yeast HSF. The EMBO Journal, 9, 4495-4501.

Schramm, F., Larkindale, J., Kiehlmann, E., Ganguli, A., Englich, G., Vierling, E., \& Von Koskull-Döring, P. (2008). A cascade of transcription factor DREB2A and heat stress transcription factor HsfA3 regulates the heat stress response of Arabidopsis. Plant Journal, 53, 264-274.

Thiele, T., Steil, L., Gebhard, S., Scharf, C., Hammer, E., Brigulla, M., ... Greinacher, A. (2007). Profiling of alterations in platelet proteins during storage of platelet concentrates. Transfusion, 47, 1221-1233.

Treuter, E., Nover, L., Ohme, K., \& Scharf, K. D. (1993). Promoter specificity and deletion analysis of three heat stress transcription factors of tomato. MGG Molecular \& General Genetics, 240, 113-125.

Vitlin, G. A., Nisemblat, S., Azem, A., \& Weiss, C. (2013). The complexity of chloroplast chaperonins. Trends in Plant Science, 18, 688-694.

Yoshida, T., Ohama, N., Nakajima, J., Kidokoro, S., Mizoi, J., Nakashima, K., ... Yamaguchi-Shinozaki, K. (2011). Arabidopsis HsfA1 transcription factors function as the main positive regulators in heat shock-responsive gene expression. Molecular Genetics and Genomics, 286, 321-332.

Zhang, L., Liu, X., Gaikwad, K., Kou, X., Wang, F., Tian, X., ... Vierling, E. (2017). Mutations in elF5B confer thermosensitive and pleiotropic phenotypes via translation defects in Arabidopsis thaliana. The Plant Cell. tpc.00808.2016

Zhu, X., Thalor, S. K., Takahashi, Y., Berberich, T., \& Kusano, T. (2012). An inhibitory effect of the sequence-conserved upstream open-reading frame on the translation of the main open-reading frame of HsfB1 transcripts in Arabidopsis. Plant, Cell and Environment, 35, 2014-2030.

\section{SUPPORTING INFORMATION}

Additional supporting information may be found online in the Supporting Information section at the end of the article.

How to cite this article: Fragkostefanakis S, Simm S, ElShershaby A, et al. The repressor and co-activator HsfB1 regulates the major heat stress transcription factors in tomato. Plant Cell Environ. 2019;42:874-890. https://doi.org/ 10.1111/pce.13434 